\title{
Maintained Representations of the Ipsilateral and Contralateral Limbs during Bimanual Control in Primary Motor Cortex
}

\author{
${ }^{\circledR}$ Kevin P. Cross, ${ }^{1}$ Ethan A. Heming, ${ }^{1}{ }^{\circledR}$ Douglas J. Cook, ${ }^{1,2}$ and ${ }^{\circledR}$ Stephen H. Scott ${ }^{1,3,4}$ \\ ${ }^{1}$ Centre for Neuroscience Studies, ${ }^{2}$ Department of Surgery, ${ }^{3}$ Department of Biomedical and Molecular Sciences and, and ${ }^{4}$ Department of Medicine, \\ Queen's University, Kingston, Ontario K7L 3N6, Canada
}

Primary motor cortex (M1) almost exclusively controls the contralateral side of the body. However, M1 activity is also modulated during ipsilateral body movements. Previous work has shown that M1 activity related to the ipsilateral arm is independent of the M1 activity related to the contralateral arm. How do these patterns of activity interact when both arms move simultaneously? We explored this problem by training 2 monkeys (male, Macaca mulatta) in a postural perturbation task while recording from M1. Loads were applied to one arm at a time (unimanual) or both arms simultaneously (bimanual). We found $83 \%$ of neurons $(n=236)$ were responsive to both the unimanual and bimanual loads. We also observed a small reduction in activity magnitude during the bimanual loads for both limbs (25\%). Across the unimanual and bimanual loads, neurons largely maintained their preferred load directions. However, there was a larger change in the preferred loads for the ipsilateral limb $(\sim 25 \%)$ than the contralateral limb $(\sim 9 \%)$. Lastly, we identified the contralateral and ipsilateral subspaces during the unimanual loads and found they captured a significant amount of the variance during the bimanual loads. However, the subspace captured more of the bimanual variance related to the contralateral limb $(97 \%)$ than the ipsilateral limb (66\%). Our results highlight that, even during bimanual motor actions, M1 largely retains its representations of the contralateral and ipsilateral limbs.

Key words: bimanual control; feedback; primary motor cortex; stable; subspace

Significance Statement

Previous work has shown that primary motor cortex (M1) represents information related to the contralateral limb, its downstream target, but also reflects information related to the ipsilateral limb. Can M1 still represent both sources of information when performing simultaneous movements of the limbs? Here we record from M1 during a postural perturbation task. We show that activity related to the contralateral limb is maintained between unimanual and bimanual motor actions, whereas the activity related to the ipsilateral limb undergoes a small change between unimanual and bimanual motor actions. Our results indicate that two independent representations can be maintained and expressed simultaneously in M1.

Received Mar. 30, 2020; revised July 15, 2020; accepted July 16, 2020.

Author contributions: K.P.C., E.A.H., and D.J.C. performed research; K.P.C., E.A.H., and S.H.S. analyzed data; K.P.C. wrote the first draft of the paper; K.P.C., E.A.H., and S.H.S. edited the paper; K.P.C. and S.H.S. wrote the paper; E.A.H. and S.H.S. designed research.

This work was supported by the Canadian Institute of Health Research. K.P.C. was supported by an Ontario Graduate Scholarship. E.A.H. was supported by a Natural Sciences and Engineering Research Council scholarship. S.H.S. was supported by a GSK chair in Neuroscience. We thank Kim Moore, Simone Appaqaq, Justin Peterson, and Helen Bretzke for laboratory and technical assistance; and members of the LIMB laboratory for constructive criticisms.

S.H.S. is co-founder and CSO of Kinarm, which commercializes the robotic technology used in the present study. The remaining authors declare no competing financial interests.

Correspondence should be addressed to Kevin P. Cross at 13kc18@queensu.ca.

https://doi.org/10.1523/JNEUROSCI.0730-20.2020

Copyright $\odot 2020$ the authors

\section{Introduction}

Motor cortex is primarily involved with controlling the contralateral side of the body. Output projections from motor cortex principally target muscles for the contralateral limb (Cheney and Fetz, 1980; Dum and Strick, 1996; Brösamle and Schwab, 1997; Lacroix et al., 2004; Rosenzweig et al., 2009; Kuypers, 2011; Soteropoulos et al., 2011) and micro-stimulation in motor cortex elicits mainly contralateral limb movements (Montgomery et al., 2013). However, activity in motor cortex is modulated by movements with either the ipsilateral or contralateral limbs (Donchin et al., 1998; Kermadi et al., 1998; Cramer et al., 1999; Ganguly et al., 2009; Diedrichsen et al., 2013; Berlot et al., 2019). Neural recordings indicate that $\sim 50 \%$ of neurons that are active for contralateral limb movements are also active for ipsilateral limb movements (Steinberg et al., 2002; Cisek et al., 2003; Heming et al., 2019). Ipsilateral-related activity also exhibits broad tuning to 
reach direction (Steinberg et al., 2002; Cisek et al., 2003) and applied loads (Heming et al., 2019).

A largely unexplored question is how motor cortex represents the limbs during bimanual movements. Many neurophysiological investigations of bimanual movements have focused on premotor areas, such as dorsal premotor and supplementary motor cortex (Tanji et al., 1987, 1988; Donchin et al., 1998; Kermadi et al., 2000; Willett et al., 2020). During unimanual reaches, these areas exhibit similar tuning for the contralateral and ipsilateral limbs (Steinberg et al., 2002; Cisek et al., 2003) with overlapping subspaces $(\sim 50 \%)$ for the contralateral- and ipsilateral-related activities (Willett et al., 2020). During bimanual motor actions, the contralateral-related activity is largely unchanged, whereas the ipsilateral activity is reduced by $\sim 50 \%$ (Rokni et al., 2003; Willett et al., 2020). It has been hypothesized that the suppression of the ipsilateral representation and its decoupling from the contralateral representation reduces its interference on the descending contralateral motor commands during bimanual control (Rokni et al., 2003; Willett et al., 2020).

However, it is unclear whether a similar change and suppression of the ipsilateral-related activity would occur in primary motor cortex (M1). During unimanual movements, M1 has decoupled representations for the contralateral and ipsilateral limbs as neurons are tuned independently for each arm (Cisek et al., 2003; Heming et al., 2019) and contralateral- and ipsilateralrelated activities occupy orthogonal subspaces (Ames and Churchland, 2019; Heming et al., 2019; Downey et al., 2020). Thus, M1 could maintain its representations of each limb across unimanual and bimanual movements as the representations are already decoupled.

We explored this hypothesis by training monkeys in a postural perturbation task. Monkeys performed this tasking using only one arm at a time (unimanual) and using both arms simultaneously (bimanual). We found that almost all neurons active during unimanual loads were also active for bimanual loads, and vice versa. There was a small reduction in the magnitude of activity related to both arms during the bimanual loads. We also found that neurons largely maintained their preferred load direction across the unimanual and bimanual loads, with a stronger relationship for the contralateral-related activity than the ipsilateral-related activity. Lastly, the contralateral and ipsilateral subspaces identified during the unimanual loads captured a significant amount of variance for the bimanual loads.

\section{Materials and Methods \\ Animals and apparatus}

Two male nonhuman primates (Macaca mulatta, weight $\sim 15 \mathrm{~kg}$ ) were trained to place their arms into an exoskeleton robot (Kinarm; Fig. 1A) (Scott, 1999) and perform a postural perturbation task similar to our previous work (Herter et al., 2009; Pruszynski et al., 2014; Heming et al., 2019). At the start of each trial, a target appeared $(0.8 \mathrm{~cm}$ diameter, red for right, blue for left, luminance matched) that was placed in front of the shoulder joint with a starting joint position of $30^{\circ}$ at the shoulder and $90^{\circ}$ at the elbow. The monkey held its hand inside the target for $500-1000 \mathrm{~ms}$, after which a load was applied by the exoskeleton that displaced the hand from the target. The monkey had $1000 \mathrm{~ms}$ to return its hand to the target and hold within the target for 1000-1500 ms to receive water reward. On a given trial, the monkey performed this task with only one hand (Fig. 1B; unimanual contexts, contralateral-only and ipsilateralonly; Fig. $1 B$ ) or both hands at the same time (Fig. $1 C$; bimanual contexts, mirror and opposite). The appearance of one or two targets at the start of the trial cued the monkey about whether one hand or both hands were required. Within a block, all unimanual and bimanual trials were randomly interleaved, and monkeys completed a minimum of 10 blocks.
A
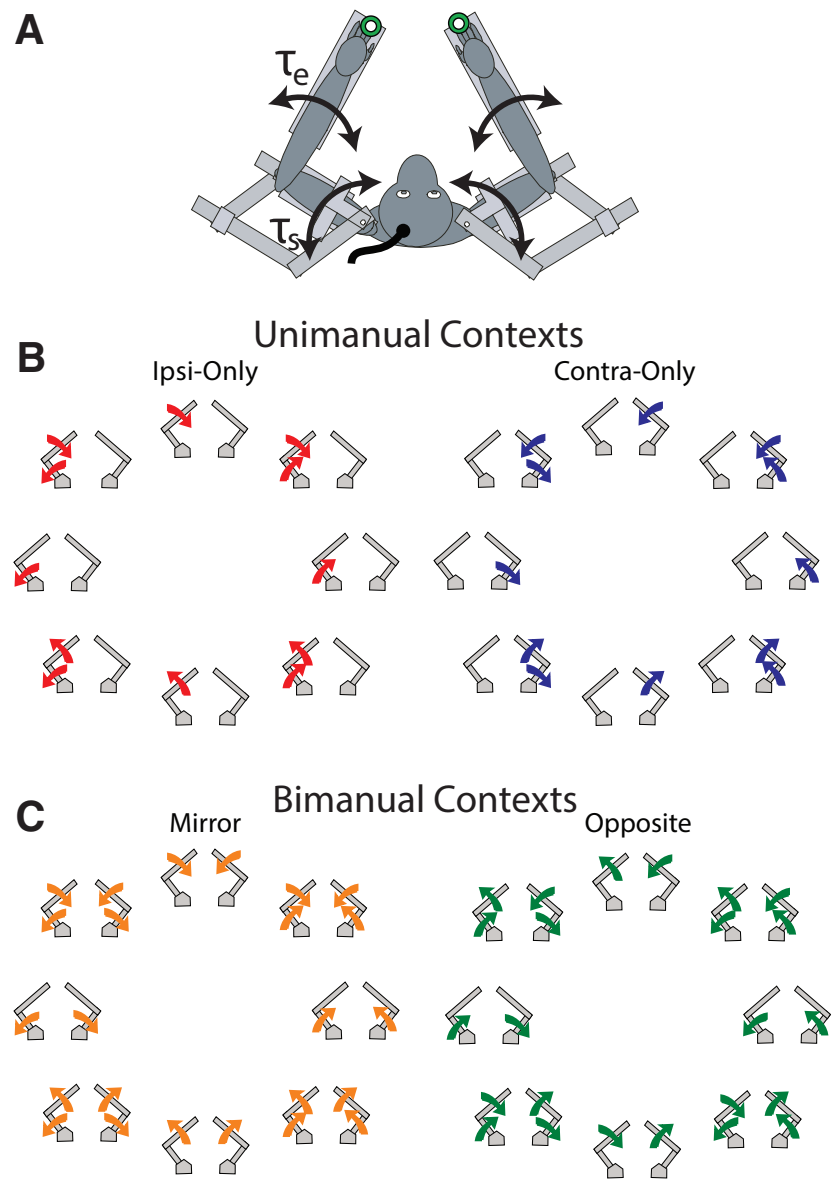

Figure 1. Experimental setup. $\boldsymbol{A}$, Monkeys were trained in a postural perturbation task using their left and/or right limbs. $\boldsymbol{B}$, For the unimanual contexts, monkeys kept either the ipsilateral or contralateral hand at a spatial target and loads were applied only to that limb. C, For the bimanual contexts, monkeys kept both hands at a spatial target and loads were applied to both limbs simultaneously. Bimanual loads were applied to the contralateral and ipsilateral limbs that were either the same magnitudes and directions (left, mirror) or the same magnitudes but opposite directions (right, opposite).

Loads consisted of flexion and extension torques applied to the shoulder and/or elbow joints. Eight combinations were used, including four single-joint torques (elbow extension [EE], elbow flexion [EF], shoulder extension [SE], and shoulder flexion [SF]) and four multijoint torques (SF/EF, SF/EE, SE/EF, SE/EE). For Monkey $\mathrm{P}$, single-joint torques consisted of $\pm 0.20 \mathrm{Nm}$ torques $(+=$ flexion load; - = extension load), whereas multijoint torques consisted of $\pm 0.14 \mathrm{Nm}$ torques applied to both joints. Monkey $\mathrm{M}$ completed this task with two different torque magnitudes: a large and a small load set. The large/small load set included single-joint torques of $\pm 0.30 / 0.20 \mathrm{Nm}$ and multijoint torques that consisted of \pm 0.24 / $0.14 \mathrm{Nm}$ torques applied to both joints.

For the bimanual loads, it was not feasible to test all possible torque combinations between the two arms. Instead, we focused on load combinations that were mirror symmetric across both arms (Fig. 1C, left; e.g., contralateral SF/EE, ipsilateral SF/EE) and load combinations that were equal in magnitude but opposite in direction (Fig. $1 C$, right; e.g., contralateral SF/EE, ipsilateral SE/EF).

Neural and kinematic recordings

Monkeys had Utah Arrays (96-channel, Blackrock Microsystems) implanted into the arm region of M1. Neural signals were digitized by a 128-Channel Neural Signal Processor (Blackrock Microsystems) at $30 \mathrm{kHz}$. An offline spike sorter (Plexon) was used to manually identify well-isolated single units by clustering waveforms in the 3 -d space spanned by the top 2 principal components of the spike waveforms and 
the peak-to-trough amplitude (Takei et al., 2018). A recent study suggests that multiunits could have been used for some of our population-level analysis below (Trautmann et al., 2019); however, we have elected to analyze only the well-isolated single units as our analysis also includes characterizing single-neuron properties. For Monkey $\mathrm{P}$, neural signals were recorded in three sessions with $\sim 4$ months between each recording session. For Monkey M, when performing the task with the small loads, neural signals were recorded from two sessions spaced 3 months apart. When performing the task with the large loads, Monkey $\mathrm{M}$ was unable to complete all 10 blocks in 1 recording day. Instead, Monkey $\mathrm{M}$ completed the 10 blocks over the course of 2 or 3 consecutive days, yielding one session. We only included single units we could isolate consistently across the recording days and had qualitatively similar spike waveforms and interspike interval histograms. Two sessions were collected that were spaced 4 months apart. The recordings with the small and large loads were separated by almost a year.

Neurons across all recorded sessions for a given monkey were treated as independent and pooled. Previously, we have estimated that $<5 \%$ of neurons would have overlapped between sessions that were spaced out by $>3$ months (Heming et al., 2019). However, recently, Gallego et al. (2020) suggested that some neurons may be recorded on chronic arrays for more than a year. We ensured that our results were unaffected by overlapping neurons across sessions by also analyzing a subset of nonoverlapping neurons. This subset was constructed by comparing which channel each neuron was recorded across sessions. If a channel recorded neurons on multiple sessions, then the neurons recorded during one session were kept and the neurons recorded on the other sessions were discarded.

Joint angles, velocities, and accelerations were also recorded by the Neural Signal Processor at $1 \mathrm{kHz}$.

\section{Data and statistical analysis}

Kinematic analysis. Kinematic signals were low-pass filtered at $10 \mathrm{~Hz}$ using a third-order Butterworth filter. We quantified the integrated and maximal hand speed over the first $300 \mathrm{~ms}$ after the perturbation (perturbation epoch) as well as the exact hand speed at the $300 \mathrm{~ms}$ time point. Statistical significance was assessed using a one-way ANOVA with load context as a factor (levels: contralateral-only, ipsilateral-only, mirror, opposite). Post hoc Tukey-Kramer tests were used to assess significance between levels.

Spike train and time epochs. The instantaneous activity of a neuron was estimated by convolving the spike time stamps with a kernel approximating a postsynaptic potential ( $1 \mathrm{~ms}$ rise and $20 \mathrm{~ms}$ fall) (Thompson et al., 1996). Activity in the perturbation epoch was calculated by aligning to the load onset and averaging across trials for the first $300 \mathrm{~ms}$. Steadystate activity was calculated by aligning to the load offset at the end of the trial and averaging across trials for the $1000 \mathrm{~ms}$ that preceded the load offset.

Dynamic range. The dynamic range was calculated to compare the relative magnitudes of the bimanual and additive model activities. During the perturbation epoch, we calculated the mean activity during the epoch for each load combination, creating eight separate values for each context. The difference between the largest and smallest mean activity within a context was defined as the dynamic range. An identical procedure was used to calculate the dynamic range in the steady-state epoch. A paired $t$ test was used to compare the activities across neurons between the bimanual loads and the appropriate additive models.

Linear model fits. Similar to our previous work, we characterized each neuron's relationship with the loads applied to the shoulder and elbow joints by fitting the activity to a linear model (Cabel et al., 2001; Herter et al., 2009; Heming et al., 2019). The mean activities for each neuron were regressed onto the applied torques to estimate tuning and magnitude. For each neuron, separate $8 \times 1$ arrays were constructed that contained the contralateral-only $\left(f r_{\text {Contra }}\right)$ and ipsilateral-only $\left(f r_{\text {Ipsi }}\right)$ activities. The mean activity of each array was subtracted and fit with the following equations:

$$
\begin{gathered}
f r_{\text {Contra }}=\alpha \tau_{C S}+\beta \tau_{C E} \\
f r_{I p s i}=\gamma \tau_{I S}+\delta \tau_{I E}
\end{gathered}
$$

where $\tau_{C S}, \tau_{C E}$ are $8 \times 1$ arrays containing the torques applied to the contralateral shoulder and elbow joints, respectively, and $\tau_{I S}, \tau_{I E}$ are $8 \times 1$ arrays containing the torques applied to the ipsilateral shoulder and elbow joints, respectively. The $\alpha, \beta, \gamma$, and $\delta$ are scalar fit parameters. For the contralateral torques, the activity magnitude of a neuron was calculated by $\sqrt{\alpha^{2}+\beta^{2}}$. The neuron's preferred direction was calculated as $\theta=\tan ^{-1} \frac{\beta}{\alpha}$, and the angle was adjusted according to the signs of $\beta$ and $\alpha\left(\alpha<0\right.$ and $\beta>0, \theta^{\prime}=\theta+180 ; \alpha>0$ and $\beta<0$, $\theta^{\prime}=\theta+360 ; \alpha<0$ and $\left.\beta<0, \theta^{\prime}=\theta+180\right)$. Similar formulas were used for the ipsilateral torques using the $\gamma$ and $\delta$ fit parameters.

For the bimanual data, we also characterized the neuron's activity related to the loads applied to the shoulder and elbow joints. However, regressing the mirror activity onto the applied loads resulted in the contralateral loads being collinear with the ipsilateral loads as the exact same loads were applied to each limb. Thus, there can be no unique solution relating the contralateral and ipsilateral torques to the neural activity. The same collinear problem also arises when regressing the loads onto the bimanual opposite activity as the contralateral and ipsilateral loads are the same up to a minus sign.

However, a unique solution could be found by concatenating the activity for the mirror $\left(f r_{\text {Mirror }}\right)$ and opposite $\left(f r_{O p p o s i t e}\right)$ contexts $\left(f r_{\text {Bimanual }}\right.$, $16 \times 1$ array). This prevents the contralateral and ipsilateral torques from being collinear. As before, the mean activity of $f r_{\text {Bimanual }}$ was subtracted and fit with the following equation:

$$
f r_{\text {Bimanual }}=\left(\begin{array}{c}
f r_{\text {Mirror }} \\
f r_{\text {Opposite }}
\end{array}\right)=\alpha\left(\begin{array}{c}
\tau_{C S}^{M} \\
\tau_{C S}^{O}
\end{array}\right)+\beta\left(\begin{array}{c}
\tau_{C E}^{M} \\
\tau_{C E}^{O}
\end{array}\right)+\gamma\left(\begin{array}{c}
\tau_{I S}^{M} \\
\tau_{I S}^{O}
\end{array}\right)+\delta\left(\begin{array}{c}
\tau_{I E}^{M} \\
\tau_{I E}^{O}
\end{array}\right)
$$

where $\tau_{C S}^{M}, \tau_{C E}^{M}, \tau_{I S}^{M}$, and $\tau_{I E}^{M}$ are the shoulder and elbow torques applied to the contralateral and ipsilateral limbs for the mirror loads, and $\tau_{C S}^{O}$, $\tau_{C E}^{O}, \tau_{I S}^{O}$, and $\tau_{I E}^{O}$ are the shoulder and elbow torques applied to the contralateral and ipsilateral limbs for the opposite loads. In our experiment, $\tau_{C S}^{M}=\tau_{C S}^{O}$ and $\tau_{C E}^{M}=\tau_{C E}^{O}$, whereas $\tau_{I S}^{M}=-\tau_{I S}^{O}$ and $\tau_{I E}^{M}=-\tau_{I E}^{O}$.

However, by using both the mirror and opposite activities the estimated fit parameters were less affected by sampling error than the equivalent unimanual fit parameters. This was a problem for comparing activity magnitudes between contexts as higher sampling error will overestimate activity magnitude (Willett et al., 2020). Consider an example where we estimate $\alpha$ with some sampling error $\eta$ such that $\hat{\alpha}=\alpha+\eta$. For simplicity, we assume $\beta$ and $\hat{\beta}$ are zero, although this is not necessary. Calculating the magnitude results in $\sqrt{\hat{\alpha}^{2}}=\sqrt{(\alpha+\eta)^{2}}=\sqrt{\alpha^{2}+2 \alpha \eta+\eta^{2}}$. Since $\eta^{2}>0$, this introduces a positive bias in our estimate of the magnitude. The term $2 \alpha \eta$ can be negative, thus reducing the impact of $\eta^{2}$. However, in simulations and our data, we still found a positive bias in the activity magnitudes.

We minimized this bias by randomly sampling half of the trials from the mirror and opposite contexts. We then trial-averaged across these samples and completed our analysis described above. We repeated this $1000 \times$ and calculated the average magnitude and preferred load direction for each neuron.

Change of tuning. We quantified how single neurons changed their tuning for the contralateral and ipsilateral limbs across the unimanual and bimanual tasks. A neuron's change in tuning was defined by the difference between its preferred directions for the unimanual and bimanual contexts. We constructed a distribution reflecting the change of tuning across the population of neurons. We quantified how unimodal this distribution was using the Rayleigh unimodal coefficient ( $\mathrm{R}$ coefficient) (Batschelet, 1981). We compared our results with a null distribution that randomly shuffled the neurons' preferred directions and calculated the resulting change in angle ("Shuffle"). The R coefficient was then calculated, and the procedure was repeated 1000 times. We also generated a 
distribution that compared the tuning change expected from independent samples within a load context ("Within-Context"). We evenly split the contralateral-only trials into two separate groups. We then calculated the change in tuning between these groups by using the same procedure as above. Probability values were calculated by findings the number of $\mathrm{R}$ coefficients from the shuffle and within-context distributions that were greater than and less than the empirical $\mathrm{R}$ coefficient, respectively. We repeated this 1000 times. A similar calculation was done using the ipsilateral-only trials.

Nonlinear modeling and Akaike's Information Criteria (AIC). Previous studies have suggested a nonlinear relationship between M1 activity and the contralateral and ipsilateral limbs (Yokoi et al., 2011; Diedrichsen et al., 2013). We addressed this by fitting the bimanual activity with models that included nonlinear interaction terms between the contralateral and ipsilateral torques as follows:

$$
\begin{gathered}
\left(\begin{array}{c}
f r_{\text {Mirror }} \\
f r_{\text {Opposite }}
\end{array}\right)= \\
\left(\begin{array}{c}
\alpha \tau_{C S}^{M}+\beta \tau_{C E}^{M}+\gamma \tau_{I S}^{M}+\delta \tau_{I E}^{M}+\varepsilon \tau_{C S}^{M} \tau_{I S}^{M}+\theta \tau_{C S}^{M} \tau_{I E}^{M}+\mu \tau_{C E}^{M} \tau_{I S}^{M}+\rho \tau_{C E}^{M} \tau_{I E}^{M} \\
\alpha \tau_{C S}^{O}+\beta \tau_{C E}^{O}+\gamma \tau_{I S}^{O}+\delta \tau_{I E}^{O}+\varepsilon \tau_{C S}^{O} \tau_{I S}^{O}+\theta \tau_{C S}^{O} \tau_{I E}^{O}+\mu \tau_{C E}^{O} \tau_{I S}^{O}+\rho \tau_{C E}^{O} \tau_{I E}^{O}
\end{array}\right)
\end{gathered}
$$

where $\varepsilon, \theta, \mu$, and $\rho$ are scalar fit parameters. We used AIC to compare the linear and nonlinear models, which balances model complexity with performance (Burnham and Anderson, 2004). Given the small number of samples (16) relative to the number of parameters in each model (linear 4, interaction 8) we applied a small sample correction to the AIC.

Demixed principal component analysis (dPCA). We applied dPCA to examine how limb-dependent and limb-independent components transfer between the unimanual and bimanual tasks (Kobak et al., 2016). Similar to Willett et al. (2020), we constructed for each neuron a trial-averaged and mean-centered tensor $x_{L C T}$, where $\mathrm{L}$ is the load combination (8 levels), $C$ is the context or laterality ( 2 levels, ipsilateral-only and contralateral-only), and $\mathrm{T}$ is time (30 levels, time points $0-300 \mathrm{~ms}$ in $10 \mathrm{~ms}$ bins). dPCA then decomposes $x_{L C T}$ into a set of marginalizations as follows:

$$
x_{L C T}=\overline{x_{L}}+\overline{x_{C}}+\overline{x_{T}}+\overline{x_{L C}}+\overline{x_{L T}}+\overline{x_{C T}}+\overline{x_{L C T}}
$$

where $\overline{x_{L}}, \overline{x_{C}}$, and $\overline{x_{T}}$ reflect the marginalizations for the load, context, and time defined as follows:

$$
\begin{aligned}
& \overline{x_{L}}=\frac{1}{N_{C} N_{T}} \sum_{C, T} x_{L C T} \\
& \overline{x_{C}}=\frac{1}{N_{L} N_{T}} \sum_{L, T} x_{L C T} \\
& \overline{x_{T}}=\frac{1}{N_{L} N_{C}} \sum_{L, C} x_{L C T}
\end{aligned}
$$

where $N_{L}, N_{C}$, and $N_{T}$ are the number of load (8), context (2), and time (30) levels. The terms $\overline{x_{L T}}, \overline{x_{C T}}, \overline{x_{L C}}$, and $\overline{x_{L C T}}$ reflect the marginalizations over the interactions terms between factors defined as follows:

$$
\begin{gathered}
\overline{x_{L T}}=\frac{1}{N_{C}} \sum_{C}\left(x_{L C T}-\overline{x_{L}}-\overline{x_{C}}-\overline{x_{T}}\right) \\
\overline{x_{C T}}=\frac{1}{N_{L}} \sum_{L}\left(x_{L C T}-\overline{x_{L}}-\overline{x_{C}}-\overline{x_{T}}\right) \\
\overline{x_{L C}}=\frac{1}{N_{T}} \sum_{T}\left(x_{L C T}-\overline{x_{L}}-\overline{x_{C}}-\overline{x_{T}}\right) \\
\overline{x_{L C T}}=x_{L C T}-\overline{x_{L}}-\overline{x_{C}}-\overline{x_{T}}-\overline{x_{L T}}-\overline{x_{C T}}-\overline{x_{L C}}
\end{gathered}
$$

we grouped marginalizations into four main categories: load-dependent $\left(\overline{x_{L}}, \overline{x_{L T}}\right)$, context-dependent $\left(\overline{x_{C}}, \overline{x_{C T}}\right)$, load-context interaction $\left(\overline{x_{L C}}, \overline{x_{L C T}}\right)$, and the time-only $\left(\overline{x_{T}}\right)$ terms.
The tensor $x_{L C T}$ was then reshaped into 1-dimensional row vector and concatenated along the rows with all recorded neurons $\left(N_{N}\right)$ yielding an $N_{N} \mathrm{x} N_{L} N_{C} N_{T}$ matrix (denoted as $X_{L C T}$ ). The same procedure was applied for all the marginalizations as well. dPCA then finds decoder $\left(D_{\varphi}\right.$, size $\mathrm{dx} N_{N}$ where $\mathrm{d}$ is the number of dimensions $\left.=10\right)$ and encoder ( $F_{\varphi}$ size $N_{N} \mathrm{xd}$ ) matrices such that the following loss function was minimized:

$$
L_{\varphi}=\left\|\overline{X_{\varphi}}-F_{\varphi} D_{\varphi} X_{L C T}\right\|
$$

where $\overline{X_{\varphi}}$ denotes the marginalization category (e.g., load-dependent). Further details of the dPCA implementation can be found in Kobak et al. (2016). We implemented dPCA using code available at https://github.com/machenslab/dPCA. We found the encoder and decoder matrices using only the activities from the unimanual tasks.

Joint optimization. We identified the unique contralateral and ipsilateral subspaces to compare how each subspace changes between the unimanual and bimanual contexts. We used a joint optimization method to identify the contralateral and ipsilateral subspaces that we have used previously (Elsayed et al., 2016; Heming et al., 2019). Briefly, this optimizer seeks a set of components for the contralateral and ipsilateral activities that maximized the amount of variance captured while constrained to keep the components orthogonal with respect to each other (Elsayed et al., 2016). The joint optimization was performed only on the activities from the contralateral-only and ipsilateral-only loads (unimanual contexts). In theory, we could have also applied principal components analysis (PCA) and achieved similar results as the joint optimization. However, we found traditional PCA generated components for the contralateral and ipsilateral subspaces that partially overlap consistent with our previous study (Heming et al., 2019). This overlap complicates the interpretation as the activity that is maintained in the ipsilateral subspace between the unimanual and bimanual tasks could simply reflect activity in the overlapping subspace. The same could be said about maintained activity in the contralateral subspace as well. In contrast, this was not a problem for the joint optimization as the contralateral and ipsilateral components were constrained to be orthogonal, thus identifying unique ipsilateral and contralateral subspaces.

We quantified how well the unimanual subspaces were aligned to the bimanual subspaces by calculating an alignment index (Elsayed et al., 2016). The alignment index was calculated according to the following:

$$
A I=\frac{\operatorname{Tr}\left(J^{T} \operatorname{Cov}_{\text {Mirror }} J\right)}{\operatorname{Tr}\left(P C_{\text {Mirror }}^{T} \operatorname{Cov}_{\text {Mirror }} P C_{\text {Mirror }}\right)}
$$

where $\operatorname{Cov}_{\text {Mirror }}$ is the mirror covariance matrix, $P C_{\text {Mirror }}$ is the top 5 principal components for the mirror activity. J was either the top 5 contralateral or top 5 ipsilateral components. The alignment index can range from 0 (indicating no overlap) to 1 (indicating complete overlap). Simply, this metric reflects the ratio between the total amount of variance captured by J with the amount of variance captured by the top 5 mirror principal components (i.e., the most variance any five linear components could capture).

One problem with calculating the alignment index is that the contralateral-related activity during the bimanual loads will automatically result in a low alignment index for the ipsilateral components since the ipsilateral subspace is orthogonal to the contralateral-related activity (Ames and Churchland, 2019; Heming et al., 2019). This problem is also present for the alignment index of the contralateral components with the bimanual activity. We mitigated this problem by first projecting the bimanual activity into the null space of the contralateral components. This removed the activity that resided in the contralateral subspace. The alignment index was then calculated between the ipsilateral components and the remaining bimanual activity. This procedure was repeated for calculating the alignment index between the contralateral components and the bimanual activity, except now the bimanual activity was projected into the null space of the ipsilateral components.

A null distribution was generated by randomly sampling subspaces that are biased by the data covariance matrix, as previously described 
(Elsayed et al., 2016). Probability values were calculated by findings the number of alignment indices from the null distribution that were greater than the empirical alignment index.

\section{Results}

\section{Kinematic results}

We trained monkeys to perform a postural perturbation task where loads were applied to either limb only (unimanual context), or both limbs, simultaneously (bimanual context). Monkey $\mathrm{P}$ was able to easily complete this task with an average success rate of $89 \%$. Monkey $M$ struggled with this task when the load magnitudes were $0.3 \mathrm{Nm}$ (large loads) with an average success rate of $51 \%$. In particular, Monkey M struggled with the multijoint bimanual loads, a problem also observed in a similar task with humans (Omrani et al., 2013). As a result, we also had Monkey M complete the same task using load magnitudes of 0.2 $\mathrm{Nm}$ (small loads) in a separate set of recording sessions. With the small loads, Monkey $\mathrm{M}$ had an average success rate of $87 \%$.

Figure $2 A$ shows Monkey P's left (ipsilateral) hand paths for all load combinations and contexts. For the first $300 \mathrm{~ms}$ after the load onset (colored circles), the hand trajectories were similar regardless of whether the ipsilateral loads were applied without (ipsilateral-only, red) or with (mirror and opposite, orange and green, respectively) an accompanying contralateral load. In contrast, when only contralateral loads (contralateral-only, blue) were applied, there was little movement observed in the left hand. Similarly, Figure $2 B$ shows the right (contralateral) hand for all load combinations and contexts. Contralateral loads evoked similar hand trajectories when accompanied without and with an ipsilateral load, whereas little motion was observed when only ipsilateral loads were applied. Examining the hand speed (Fig. 2C,D) revealed similar observations. The loads applied to the left arm evoked larger motion than for the contralateral limb for Monkey $\mathrm{P}$ that may reflect increased training with the right arm. This was observed to a lesser extent in Monkey M (data not shown).

We calculated the integrated hand speed over the first $300 \mathrm{~ms}$ for all load contexts (Fig. $2 E$ for Monkey $P$ and Fig. $2 G$ for Monkey M large loads). For the left hand, a one-way ANOVA with load context as a factor revealed a significant main effect for both monkeys (Monkey P: $F_{(3,28)}=35, p<0.001$; Monkey M: $\left.F_{(3,28)}=42, p<0.001\right)$. Post hoc analysis confirmed that contralateral-only loads evoked smaller hand motion in both monkeys (Fig. 2E,G, left columns). Similar results were found when we examined the maximum hand speed within the first $300 \mathrm{~ms}$ (middle column), as well as the hand speed at $300 \mathrm{~ms}$ (right column).

For the right hand, a one-way ANOVA revealed a significant main effect for the integrated hand speed for both monkeys (Monkey P: $F_{(3,28)}=35, p<0.001$; Monkey M: $F_{(3,21)}=24$, $p<0.001)$. Post hoc analysis confirmed that ipsilateral-only loads evoked smaller hand motion in both monkeys (Fig. 2F,H). Similar results were found when we examined the maximum hand speed within the first $300 \mathrm{~ms}$ (middle column) as well as the hand speed at $300 \mathrm{~ms}$ (right column). Similar results were also found when we examined Monkey M's kinematics for the smaller loads (data not shown).

\section{Neural recordings}

We recorded 92 neurons from Monkey P. From Monkey M, we recorded 66 neurons with the large loads and 78 neurons with the small loads. For Monkey M, we pooled all neurons (144) recorded for the large and small loads as our findings were similar when we analyzed each group separately.

Figure $3 A$ shows the activity of an example neuron when ipsilateral-only and contralateral-only loads were applied (top panels). For simplicity, we only present the neuron's activity for two of the multijoint loads (SF/EE light colors, SE/EF dark colors). For both contexts, this neuron displayed clear selectivity for the loads, with greater activity during ipsilateral-only loads for SE/ EF (Fig. 3A, left), and greater activity during contralateral-only loads for SF/EE (Fig. $3 A$, right). However, for the mirror context this neuron exhibited little selectivity for the loads (Fig. $3 A$, middle left). For comparison, we calculated the expected mirror activity if it simply reflected the addition of the ipsilateral-only and contralateral-only activities (additive mirror model; Fig. 3A, bottom left). The additive mirror model also showed little selectivity for the loads. For the opposite context, this neuron exhibited clear selectivity for the loads (Fig. $3 A$, middle right) and was qualitatively similar to the equivalent additive model (Fig. $3 A$, bottom right panel). Figure $3 B, C$ shows the activities for two additional example neurons.

We investigated whether a separate population of neurons were active during the unimanual and bimanual contexts. For the ipsilateral-only and contralateral-only contexts, we regressed each neuron's activity onto the ipsilateral and contralateral loads, respectively. For the bimanual contexts, we concatenated the mirror and opposite contexts and regressed the concatenated activity onto the ipsilateral and contralateral loads. This concatenation was vital as regressing the mirror and opposite contexts separately would result in the ipsilateral loads being collinear to the contralateral loads. Consistent with our previous report (Heming et al., 2019), more neurons had significant fits for the contralateral-only context (Monkey P/Monkey M, 87\%/76\%, $n=80 / 109$, total neurons $=92 / 144)$ than ipsilateral-only context (58\%/61\%,n=53/88) during the perturbation epoch (Fig. 4A,C). We also found a strong overlap between neurons with significant fits for the bimanual and unimanual contexts. For Monkey P/ Monkey M, 91\%/74\% ( $n=86 / 114)$ of neurons had significant fits for the bimanual contexts and at least one of the unimanual contexts (shaded regions). Seven percent/12\% (6/17) of neurons had significant fits for the unimanual loads only, whereas $2 \% / 5 \%$ (2/ 7) of neurons had a significant fit for the bimanual loads only. A similar overlap was observed when we examined the steady-state activity (Fig. 4B,D).

Next, we investigated whether activity during the bimanual contexts exhibited any suppression relative to the unimanual context. In the perturbation epoch, we estimated each neuron's dynamic ranges for the mirror and opposite load contexts and compared it with the dynamic ranges from the equivalent additive models. For Monkey P/Monkey M, we found the additive mirror model overestimated the activity of $78 \% / 83 \%$ of neurons (Fig. 5A,E), while the additive opposite model overestimated $61 \% / 83 \%$ of neurons (Fig. $5 B, F$ ). Across the population, the additive mirror model significantly overestimated the mirror context by $13 \% / 36 \%$ (Fig. $5 C, G$; paired $t$ test; Monkey P: $t_{(91)}=7.4$, $p<0.001$, Monkey M: $\left.t_{(143)}=11.3, p<0.001\right)$, whereas the additive opposite model overestimated the opposite context by $14 \% /$ $18 \%$ (Monkey P: $t_{(91)}=2.0, p=0.047$; Monkey M: $t_{(143)}=10.3$, $p<0.001)$. We found a similar overestimation by the additive model when we examined the steady-state epoch (Fig. 5D, H; mirror: Monkey P: $t_{(91)}=9.0, p<0.001$; Monkey M: $t_{(143)}=8.4$, $p<0.001$; opposite: Monkey P: $t_{(91)}=5.7, p<0.001$; Monkey M: $\left.t_{(143)}=9.8, p<0.001\right)$. For Monkey $\mathrm{M}$, there appears to be greater suppression at higher activities for the opposite loads, 


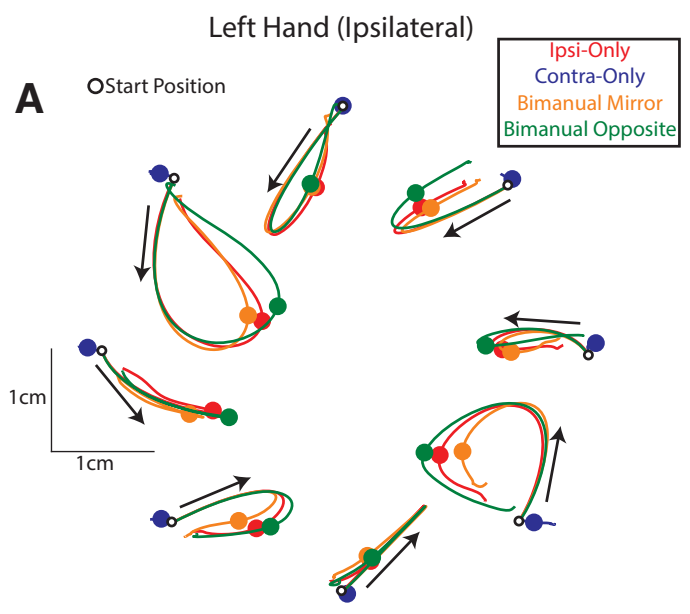

B

Right Hand (Contralateral)

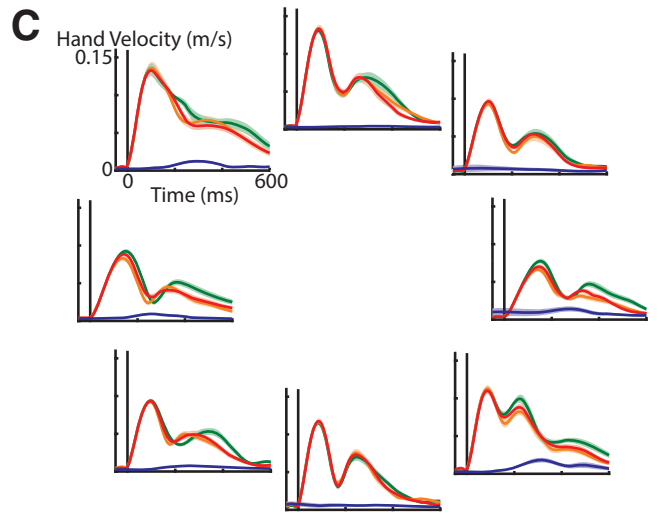

D
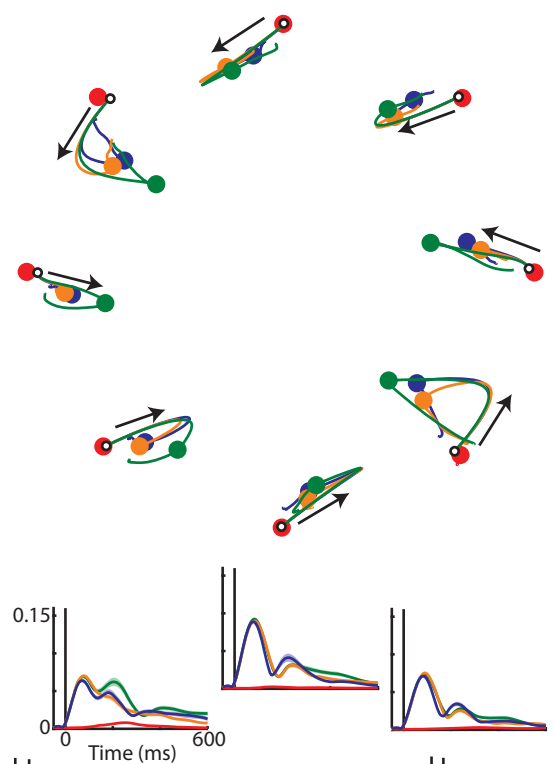

$\mathbf{E}$

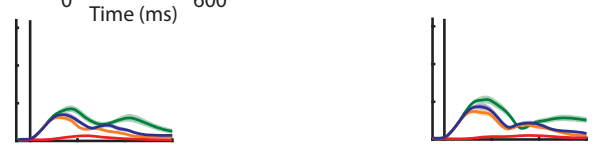

Monkey $\mathrm{P}$
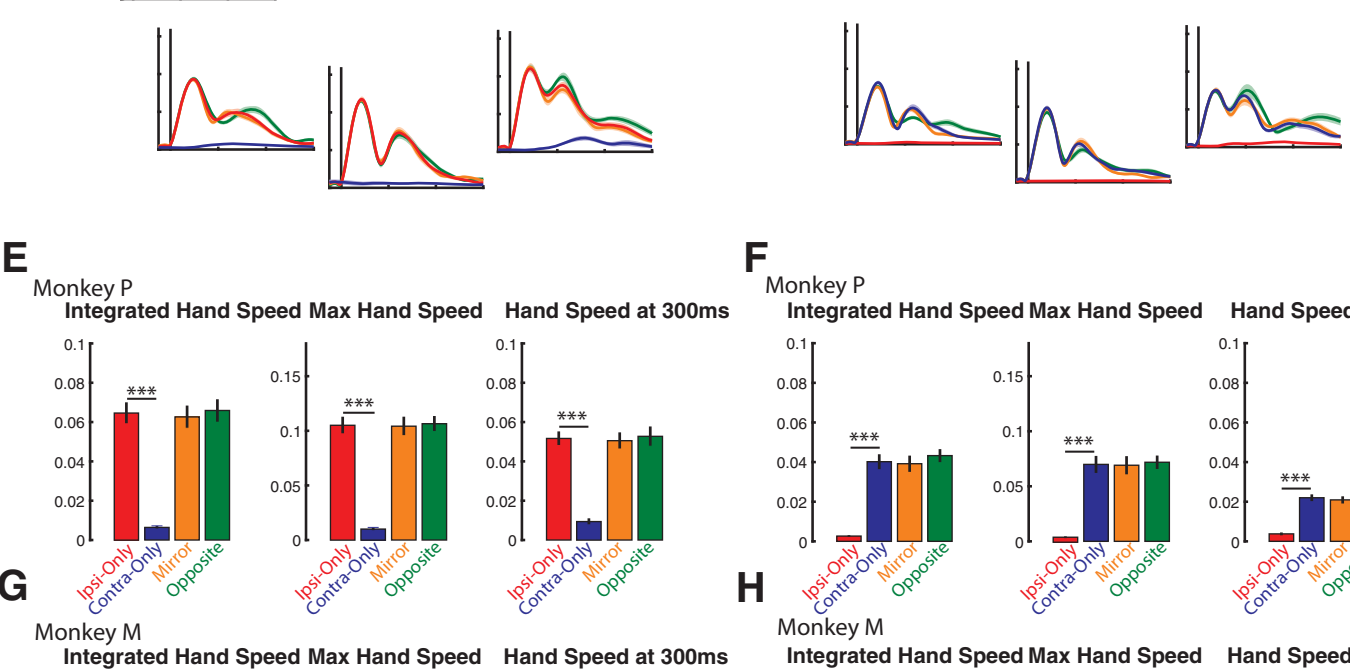

Hand Speed at $300 \mathrm{~ms}$

\section{$\mathbf{F}$}
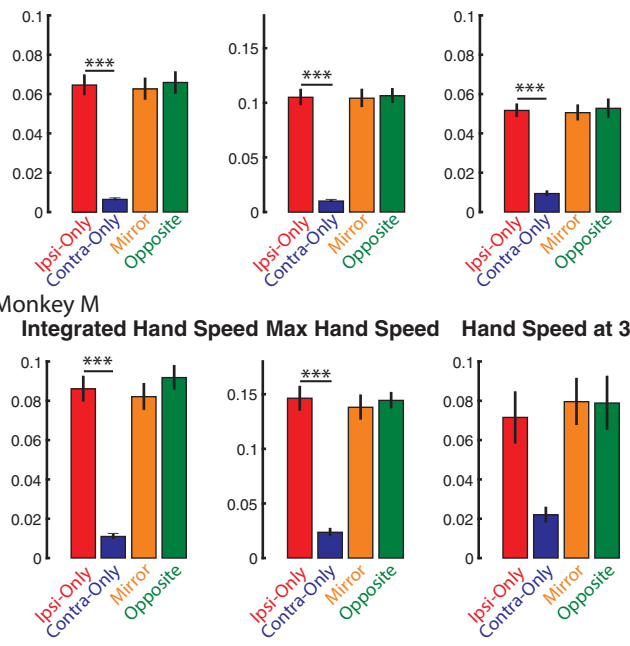

H
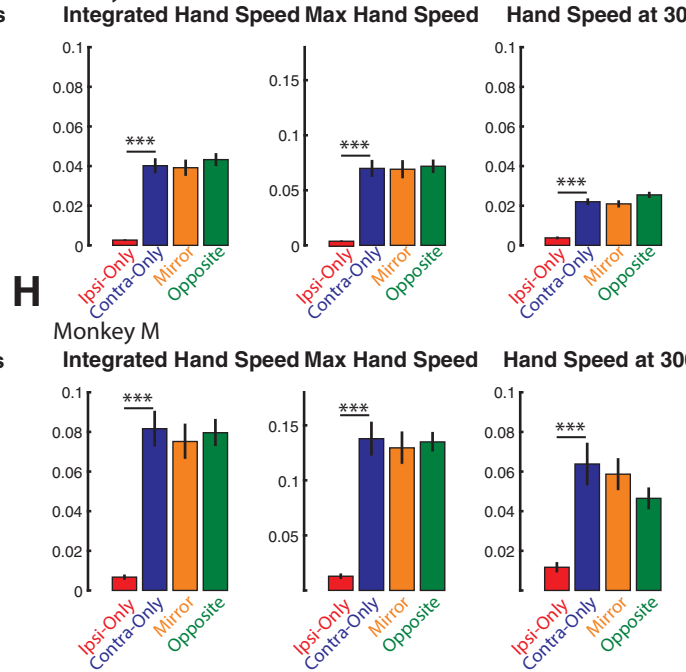

Figure 2. Hand kinematics. $A, B$, Hand paths for the left and right hand of Monkey $P$ for all load contexts. Circles represent the 300 ms mark on the hand trajectory. Arrows indicate the initial direction of motion. $\boldsymbol{C}, \boldsymbol{D}$, Left- and right-hand speeds for each perturbation type from Monkey P. $\boldsymbol{E}$, For the left hand of Monkey $P$, the mean across load combinations for the integrated hand speed, maximum hand speed, and hand speed at $300 \mathrm{~ms}$. A one-way ANOVA with context as a factor revealed a significant main effect for the integrated hand speed $\left(F_{(3,28)}=35\right.$, $p<0.001)$, maximum hand speed $\left(F_{(3,28)}=48, p<0.001\right)$, and the hand speed at $300 \mathrm{~ms}\left(F_{(3,28)}=30, p<0.001\right)$. $\boldsymbol{F}$, Same as in $\boldsymbol{E}$, but for the right hand of Monkey $P$. A significant main effect was found for the integrated hand speed $\left(F_{(3,28)}=35, p<0.001\right)$, maximum hand speed $\left(F_{(3,28)}=26, p<0.001\right)$, and the hand speed at $300 \mathrm{~ms}\left(F_{(3,28)}=41, p<0.001\right)$. G, Same as in $\boldsymbol{E}$, but for Monkey M. A significant main effect was found for the integrated hand speed $\left(F_{(3,28)}=42, p<0.001\right)$, maximum hand speed $\left(F_{(3,28)}=41 p<0.001\right)$, and the hand speed at $300 \mathrm{~ms}$ $\left(F_{(3,28)}=6, p<0.001\right)$. $\boldsymbol{H}$, Same as in $\boldsymbol{F}$, but for Monkey M. A significant main effect was found for the integrated hand speed $\left(F_{(3,28)}=24, p<0.001\right)$, maximum hand speed $\left(F_{(3,28)}=27\right.$, $p<0.001)$, and the hand speed at $300 \mathrm{~ms}\left(F_{(3,28)}=10, p<0.001\right)$. $\boldsymbol{E}-\boldsymbol{H}$, Post hoc Tukey-Kramer tests were used to compare either the unimanual ipsilateral loads $(\boldsymbol{E}, \boldsymbol{G})$ with the other three contexts or the unimanual contralateral loads with the other three contexts $(\boldsymbol{F}, \boldsymbol{H}) .{ }^{* * *} p<0.001$. All $p$ values were Bonferroni corrected with a factor of 3 . 

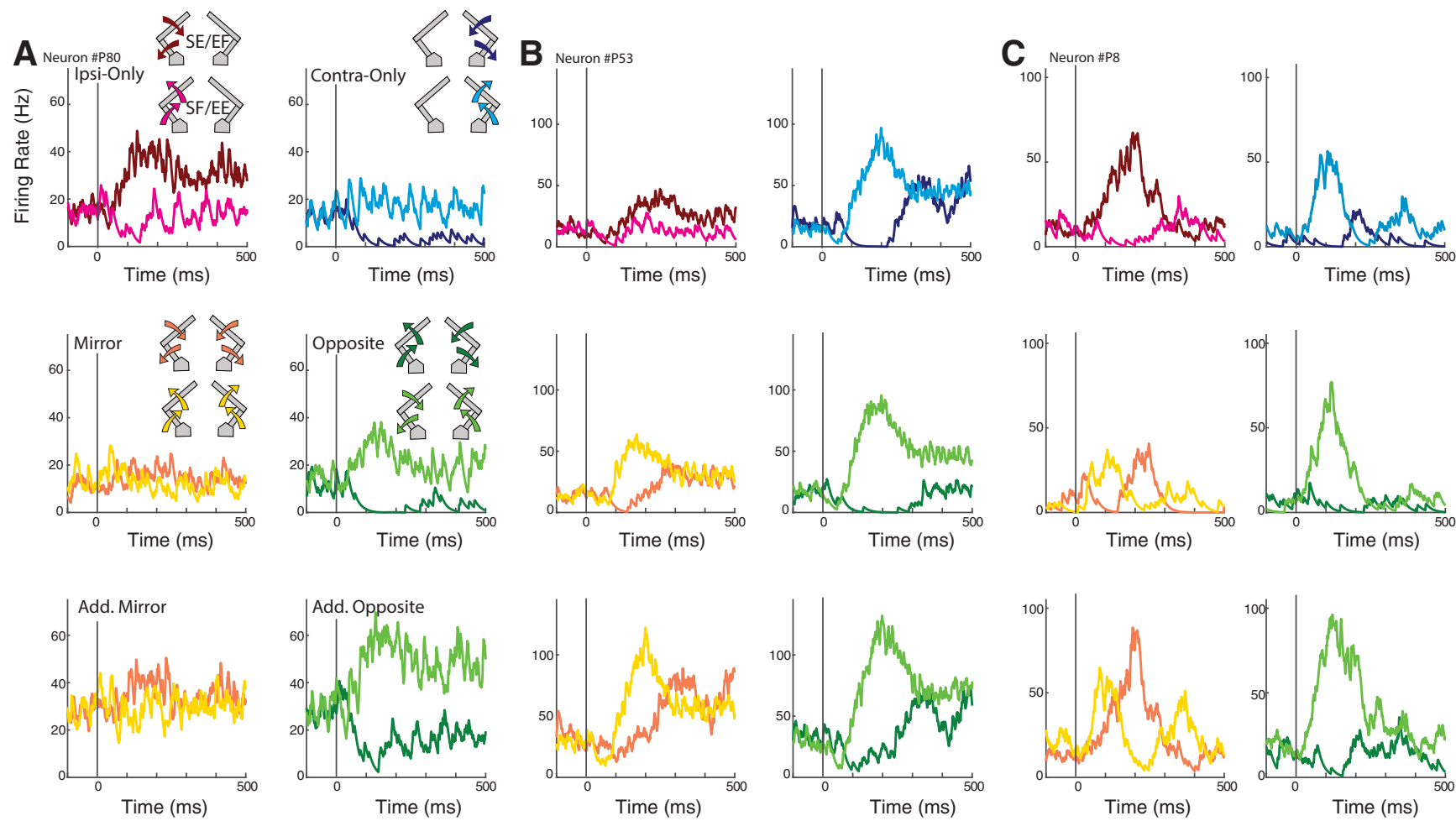

Figure 3. Activities of three example neurons. A, The activities of an example neuron for each load context. Top row, The neuron's activities for loads applied to the ipsilateral (left ipsilateral-only) and contralateral (right contralateral-only) limbs only. For simplicity, only the loads for SE/EF (dark colors) and SF/EE (light colors) are shown. Middle row, The neuron's activities for the mirror (left) and opposite (right) loads. Bottom row, The expected activities of the neuron if the mirror and opposite activities reflected a linear sum of the contralateral-only and ipsilateral-only activities. $\boldsymbol{B}, \boldsymbol{C}$, Activities from two additional example neurons.

which is absent for Monkey $\mathrm{P}$ (Fig. 5F). We verified that this group of neurons reflected a mixture of neurons recorded during the "large" and "small" load conditions (data not shown).

We explored whether the reduction in dynamic range was because of a specific suppression of the ipsilateralrelated activity. From the tuning fits, we could separate the activities related to each limb during the bimanual context and calculate the activity magnitudes (see Materials and Methods). Figure 6 compares the magnitudes between the unimanual and bimanual contexts for the contralateral- and ipsilateral-related activities. We included only neurons with significant fits for both unimanual contexts and the bimanual context (Monkey P/Monkey M: $n=41 / 69)$. In the perturbation epoch, we found that the ipsilateral-related activity was smaller during the bimanual context than the unimanual context for $80 \% / 65 \%$ of neurons in Monkey $\mathrm{P} /$ Monkey M (Fig. 6A,E). Across the population, the ipsilateral-related activity during the bimanual context was $70 \% / 82 \%$ of the size during the unimanual context for Monkey P/Monkey $\mathrm{M}$ (Monkey P: paired $t$ test: $t_{(40)}=4.9, p<0.001$; Monkey M: $t_{(68)}$ $=4.1, p<0.001)$. For the contralateral-related activity of Monkey $\mathrm{P}$, we found the magnitudes of the unimanual and for Monkey M.
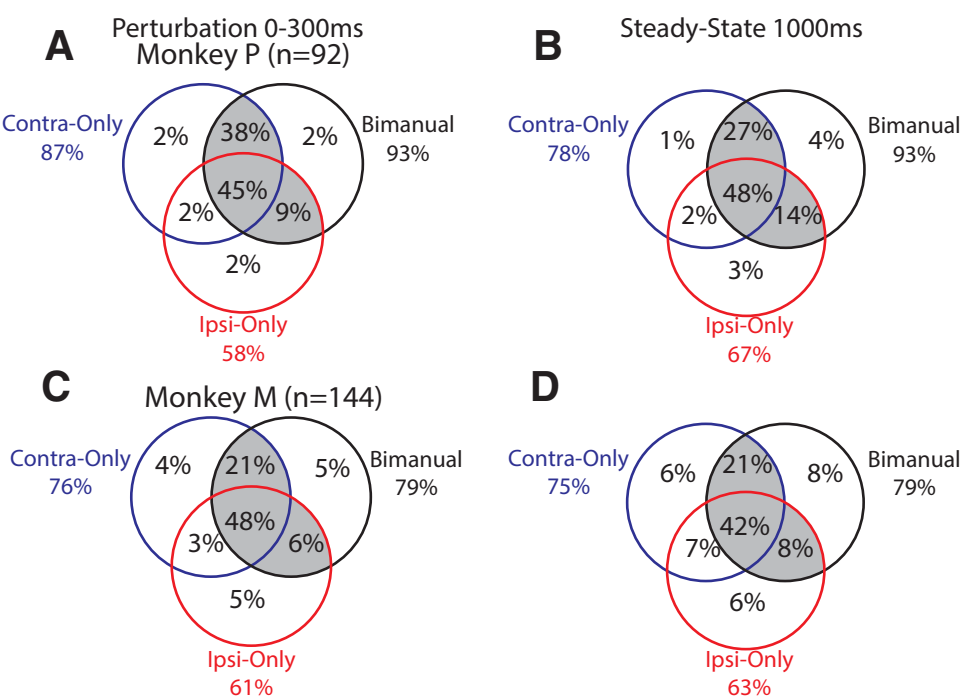

D

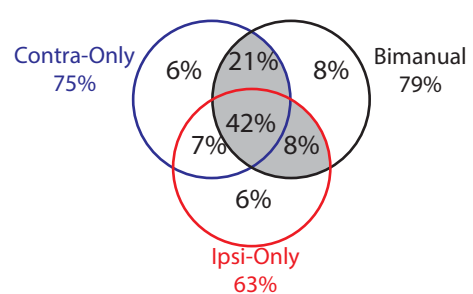

Figure 4. Neuron classification for each load context. $\boldsymbol{A}$, For Monkey $\mathrm{P}$, a Venn diagram showing the overlap between neurons with significant fits for the contralateral-only, ipsilateral-only and bimanual contexts. Values are percentages of the total population. Shaded region represents the neurons with significant fits for the bimanual contexts and at least one of the unimanual contexts (contralateral-only, ipsilateral-only). $\boldsymbol{B}$, Same as in $\boldsymbol{A}$, but for the steady-state epoch. $\boldsymbol{C}, \boldsymbol{D}$, Same as in $\boldsymbol{A}, \boldsymbol{B}$, but

bimanual contexts were similar with almost equal number of neurons residing above and below the unity line (Fig. 6B). For Monkey $\mathrm{M}$, the contralateral-related activities were smaller during the bimanual context than the unimanual context for $91 \%$ of neurons (Fig. $6 F$ ). Across the population, the contralateral-related activity during the bimanual context was $79 \%$ 

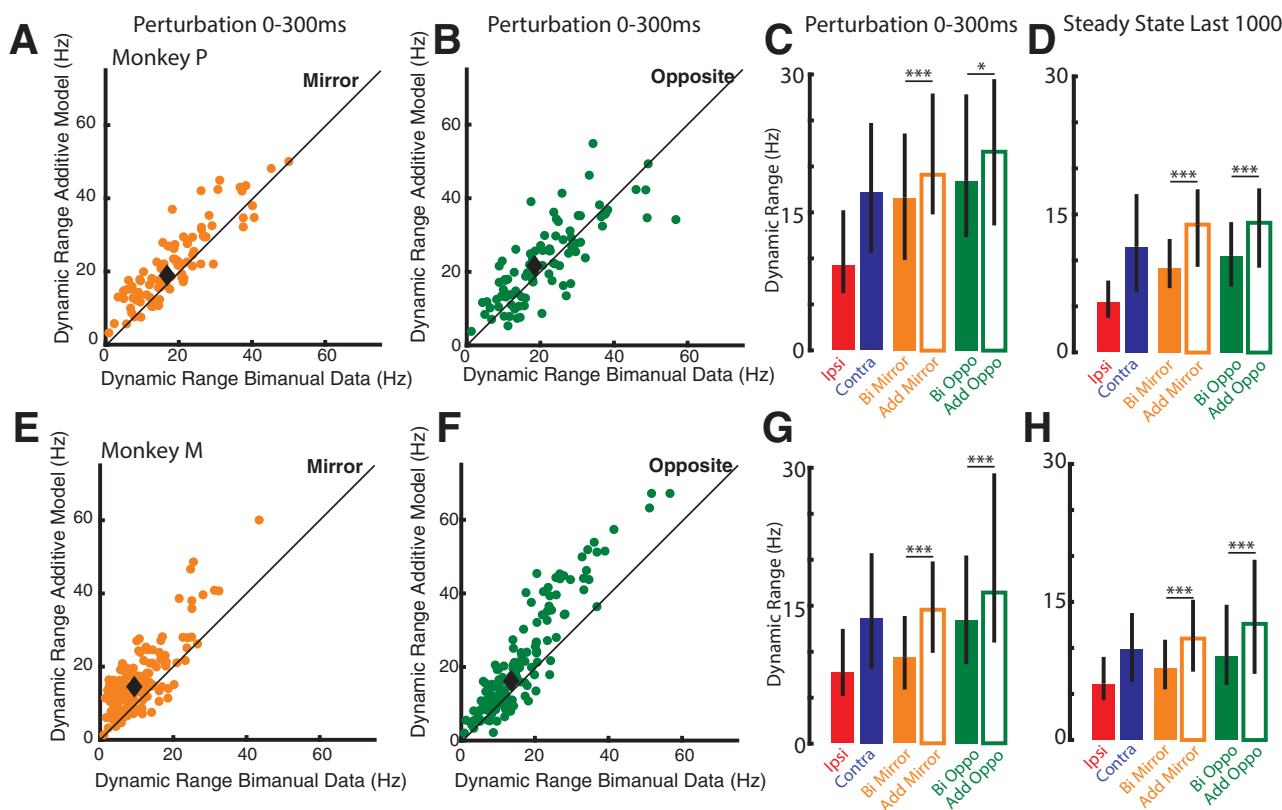

Figure 5. Dynamic range across neurons for the mirror and opposite contexts. $\boldsymbol{A}$, For Monkey $\boldsymbol{P}$, comparison between each neuron's observed dynamic range (abscissa) with its dynamic range predicted by the additive model (ordinate) for the mirror perturbations. Black diamond represents the median. $\boldsymbol{B}$, Same as in $\boldsymbol{A}$, but for the opposite context. $\boldsymbol{C}$, The median dynamic range in the perturbation epoch across all recorded neurons. Error bars indicate the 25th and 75th percentiles. $\mathbf{D}$, Same as in $\mathbf{C}$, but for the steady-state epoch. $\boldsymbol{E}$ - $\boldsymbol{H}$, Same as in $\mathbf{A}-\mathbf{D}$, but for Monkey M. ${ }^{*} p<0.05 .{ }^{* *} p<0.001$.
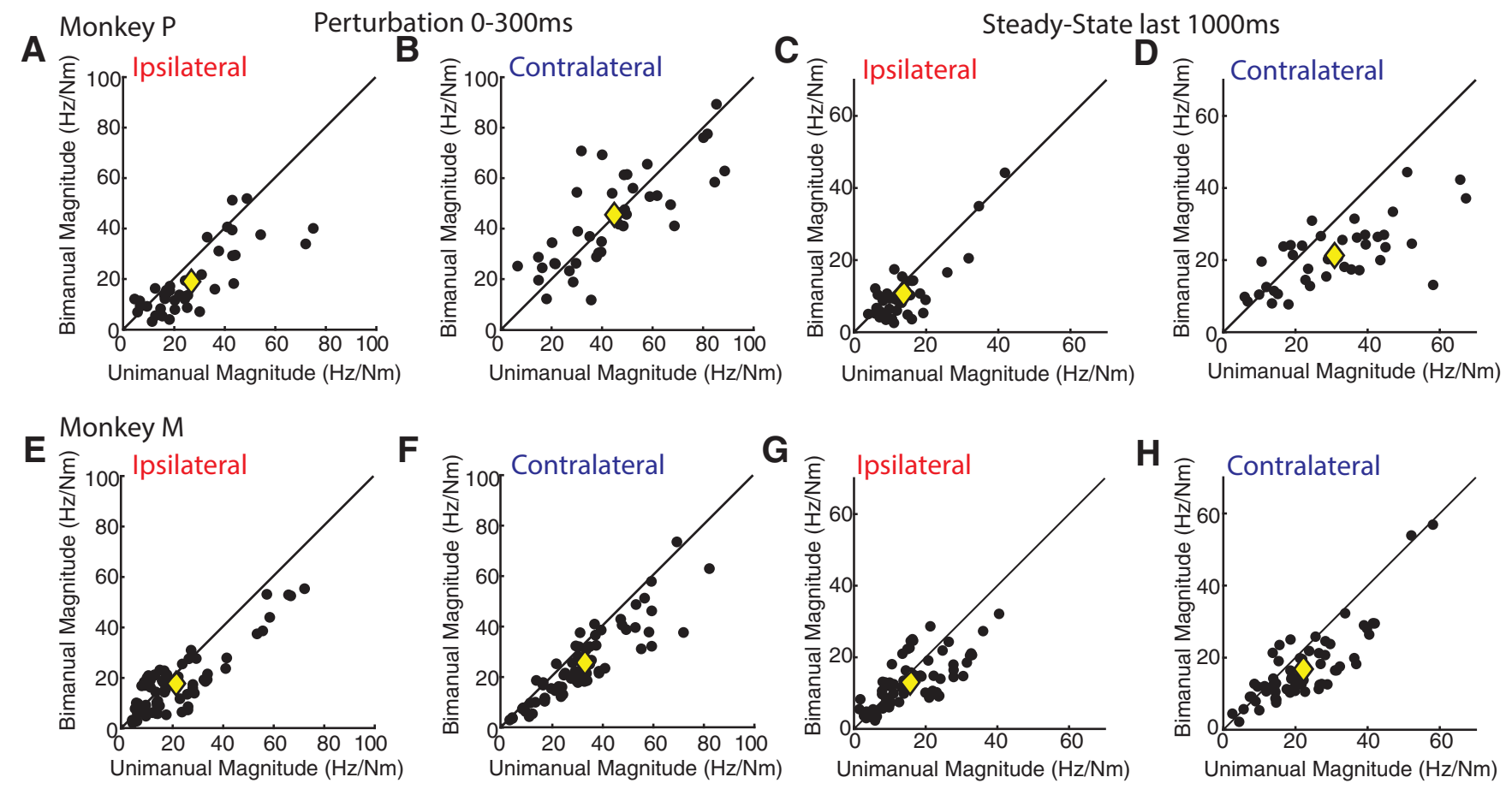

Figure 6. Magnitude changes between the unimanual and bimanual contexts. $\boldsymbol{A}$, For Monkey $\boldsymbol{P}$, comparison of the ipsilateral-related magnitudes between the unimanual (abscissa) and bimanual contexts (ordinate) during the perturbation epoch. Yellow diamond represents the median. $\boldsymbol{B}$, Same as in $\boldsymbol{A}$, but for the contralateral-related magnitudes. $\boldsymbol{C}, \boldsymbol{D}$, Same as in $\boldsymbol{A}, \boldsymbol{B}$, but for the steady-state epoch. $\boldsymbol{E}-\boldsymbol{H}$, Same as in $\boldsymbol{A}-\boldsymbol{D}$, but for Monkey M.

of the size during the unimanual context $\left(t_{(68)}=8.1, p<0.001\right)$. Examining the steady-state activity yielded similar findings (Fig. $6 C, D, G, H)$. For both monkeys, we found that the activity magnitudes were significantly reduced during the bimanual context for the ipsilateral-related (Monkey P: relative size of bimanual to unimanual: $78 \%, t_{(38)}=3.8, p<0.001$; Monkey M: $83 \% t_{(65)}=$ 3.2, $p=0.002$ ) and contralateral-related activities (Monkey P:
$69 \%, t_{(38)}=5.1, p<0.001 ;$ Monkey $\mathrm{M}: 80 \%, t_{(65)}=7.0$, $p<0.001)$. These data suggest the ipsilateral- and contralateralrelated activities exhibited roughly similar levels of suppression.

Next, we investigated whether the representations changed between unimanual and bimanual contexts. From the tuning fits, we could estimate each neuron's preferred direction for each limb during the unimanual and bimanual contexts. Figure 7 
Monkey P

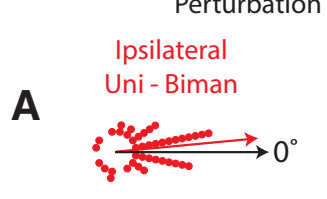

Contralateral Uni - Biman

B

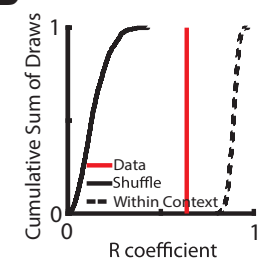

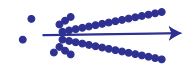

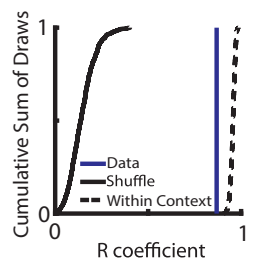

Steady-State $1000 \mathrm{~ms}$

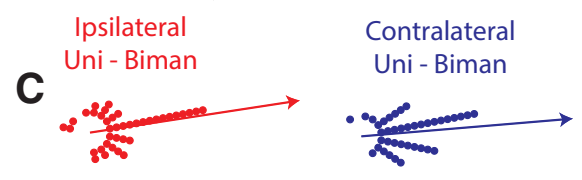

D
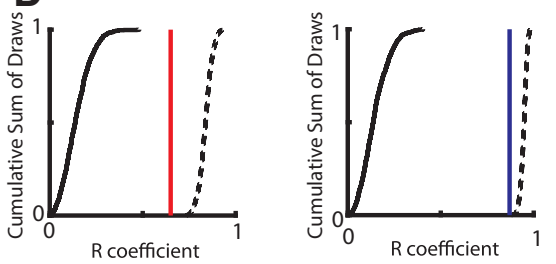

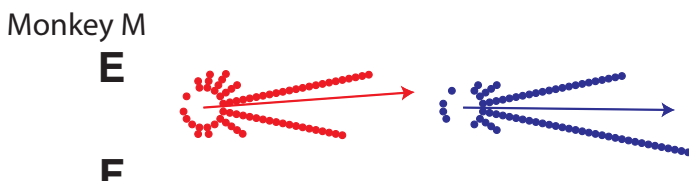

$\mathbf{F}$
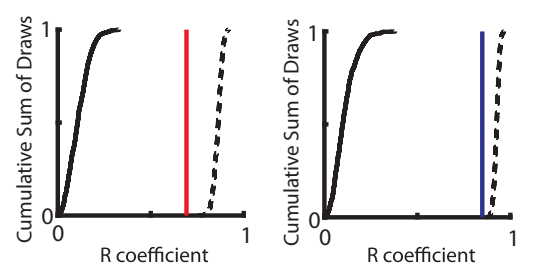
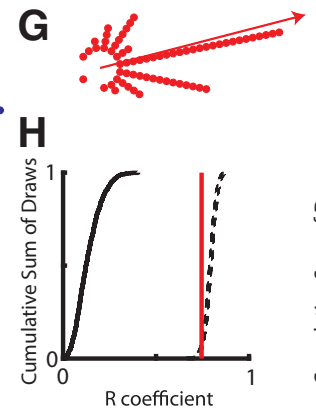
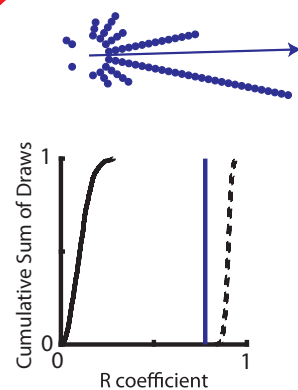

Figure 7. Change of tuning between unimanual and bimanual contexts. $\boldsymbol{A}$, Polar histograms represent the change in tuning between the unimanual and bimanual contexts for the ipsilateral (left) and contralateral loads (right) during the perturbation epoch. Neurons with no change would lie along the $0^{\circ}$ axis. $B$, Cumulative sum of Rayleigh coefficients for the observed data (blue and red lines), the shuffled distribution (black solid line), and the within-context distribution (black dashed line). $\boldsymbol{C}, \boldsymbol{D}$, Same as in $\boldsymbol{A}, \boldsymbol{B}$, but for the steady-state epoch. $\boldsymbol{E}-\boldsymbol{H}$, Same as in $\boldsymbol{A}-\boldsymbol{D}$, but for Monkey M.

displays the change in tuning between the unimanual and bimanual contexts. In the perturbation epoch, we found that the distribution for the ipsilateral-related activity was centered near the $0^{\circ}$ axis, indicating that most neurons had similar tuning for the unimanual and bimanual contexts (Fig. $7 A, E$, left). We quantified how unimodal the distribution was by calculating the Rayleigh (R) coefficient (Fig. 7B,F). For comparison, we generated a null distribution where we calculated the change in tuning after shuffling the neurons' preferred load directions (Fig. $7 B, F$, "shuffle" solid black line). We also generated a distribution that compared the tuning changes expected from two independent samples from the same context ("within context" dashed line). For the ipsilateral-related activity, the change in tuning across contexts was significantly more unimodal (red line, Monkey P/ Monkey M, Rayleigh coefficient, $\mathrm{R}=0.64 / 0.70$ ) than sampling from a shuffled distribution (both monkeys: $p<0.001$ ). However, the change in tuning was significantly less unimodal than the within-context distribution (median $\mathrm{R}=0.89 / 0.86$, $p<0.001$ ), although the difference was small (change in $\mathrm{R}$ from additive model $=28 / 19 \%$ ). We found similar results when we examined the steady state (Fig. 7C,D,G,H).

For the contralateral-related activity, we also found that the distribution was centered near the $0^{\circ}$ axis (Fig. $7 A, E$, right) and found it was significantly more unimodal (Fig. $7 B, F$, right, blue line; Monkey $\mathrm{P} /$ Monkey $\mathrm{M}: \mathrm{R}=0.87 / 0.85)$ than sampling from a shuffled distribution $(p<0.001)$. However, the change in tuning was significantly less unimodal than the within-context distribution (median $\mathrm{R}=0.96 / 0.93 p<0.001$ ), although the difference was also small (9\%/9\% change from additive model). We found similar results when we examined the steady state (Fig. $7 C, D, G, H$ ).
Next, we investigated the temporal correlations between the activities for the bimanual contexts and the additive models. We restricted our analysis to neurons that responded to both unimanual and bimanual contexts. Figure 8 shows the temporal correlations between the responses during the bimanual contexts and the equivalent additive models across all neurons. There is a clear shift in the cumulative sum toward positive correlation coefficients for both the mirror (median coefficient: Monkey $\mathrm{P} /$ Monkey M: $0.63 / 0.45)$ and opposite (0.63/0.79) contexts. As a comparison, we generated a shuffled distribution where correlations were calculated between randomly selected pairs of bimanual and additive responses (Fig. 8, insets). We found the median temporal correlations were significantly greater than the null distributions (median range: $-0.02,0.02, p<0.001$ for all comparisons). These results indicate that neurons had significant temporal patterns that were conserved between the unimanual and bimanual contexts.

\section{Population analysis}

A recent study has demonstrated limb-dependent and limb-independent activity patterns in M1 at the population level (Willett et al., 2020). Here, we explored how limb-dependent and limbindependent components transfer between the unimanual and bimanual contexts. We used dPCA (Kobak et al., 2016) to identify neural components related to limb-dependent and limb-independent activity patterns. The limb-independent components were composed of activity patterns that varied across the loads only (load) or patterns that varied across time only (time), whereas the limb-dependent components were composed of patterns that varied with the laterality of the movement (laterality) 

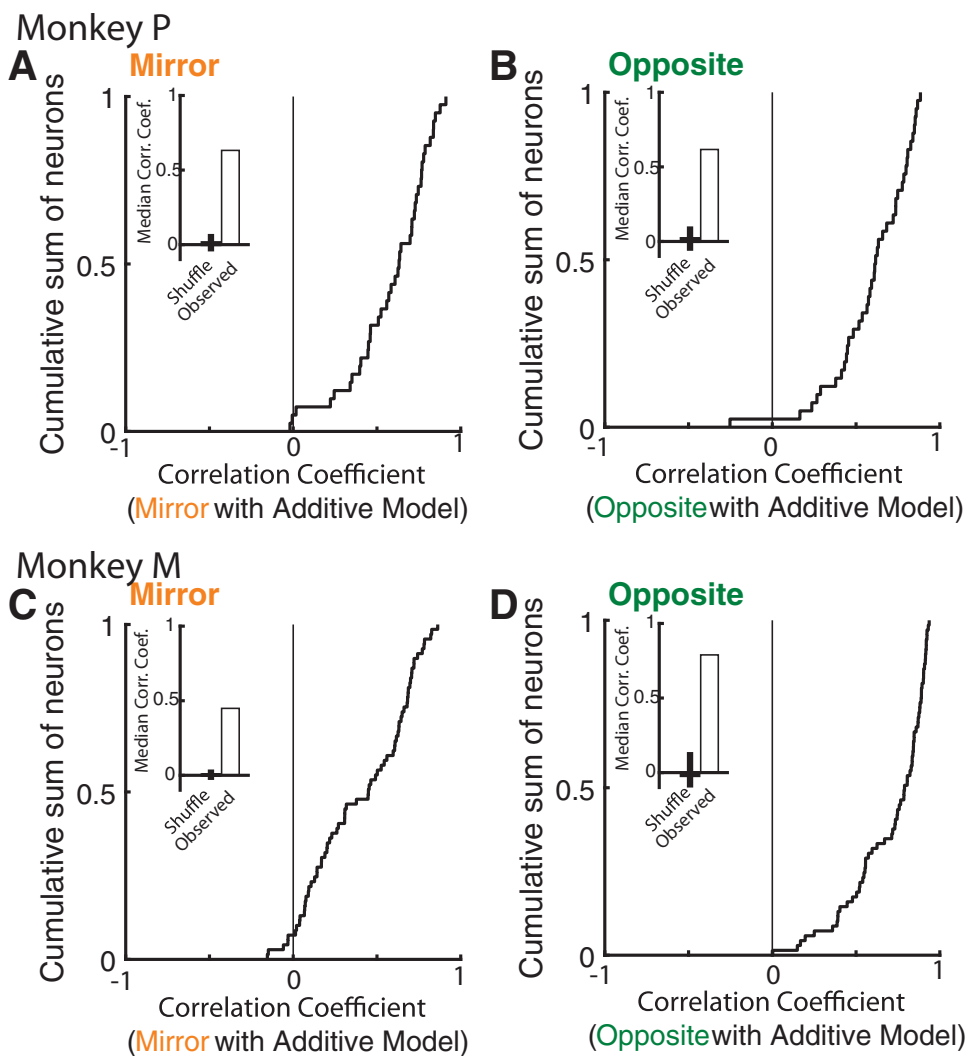

Figure 8. Temporal correlation between bimanual and additive responses. $\boldsymbol{A}$, For Monkey $P$, cumulative sum of the temporal correlation coefficients between the mirror activities and the additive mirror model. Inset, The median correlation coefficient from the sample distribution (mean \pm SD) and the observed median coefficient. $\boldsymbol{B}$, Same as in $\boldsymbol{A}$, but for the opposite loads. $\boldsymbol{C}, \boldsymbol{D}$, Same as in $\boldsymbol{A}, \boldsymbol{B}$, but for Monkey M.

or the load-laterality interaction (load/laterality). We applied dPCA using the activities related to the unimanual task and found that the two components that captured the largest amount of variance were limb-dependent (Fig. 9A,B, purple and open bars; Monkey P/Monkey M: 38\%/37\%). This is consistent with our previous work showing activity related to the ipsilateral and contralateral limbs reside in largely orthogonal subspaces and thus limb-dependent (Heming et al., 2019). The largest load-dependent components for Monkey $\mathrm{P}$ were components 3 and 4 and explained $14 \%$ of the variance (Fig. $9 A$, black bar), whereas for Monkey $M$ the largest load-dependent components were components 3 and 5 and explained $10 \%$ of the variance (Fig. $9 B$ ). Interestingly, unlike previous studies in reaching and grasping (Kaufman et al., 2016; Gallego et al., 2018; Rouse and Schieber, 2018; Willett et al., 2020), the largest time component captured only a small portion of the total variance for both monkeys (Fig. $9 A, B$, gray bar; Monkey P/Monkey M: 6\%/4\%).

Next, we examined how well these components captured the bimanual-related activity. We projected the bimanual and additive model activities onto each component. The top laterality component captured a large amount of the unimanual variance (Fig. 9A; component 1, 9B component 2, open bar) but captured only a small amount of the variance for the bimanual contexts (Fig. 9C-F, left panels) and additive models (Fig. 9C-F, right panels). This could be explained by examining the dynamics in the component (Fig. 9G,H, for simplicity, SF/EE and SE/EF loads only shown). During the contralateral-only and ipsilateral-only loads, the activities moved to two approximately equal values that were opposite in magnitude (Fig. 9H, left). As a result, during the bimanual contexts (middle and left panels, solid lines), the activities related to the contralateral and ipsilateral loads effectively cancelled each other out, leaving no activity. The same process happened for the additive models as well (dashed lines). A similar process could explain why the top load/laterality interaction component (Fig. $9 A$, component 2 ; Fig. $9 B$, component 1 , purple bar) captured a small amount of the variance for the mirror context and additive mirror model (Fig. 9C,D) but captured a large amount of the variance for the opposite context and additive opposite model (Fig. $9 E, F)$. For the contralateral-only loads (Fig. 9I, left, blue traces), the dynamics in these components separate with larger (dark blue) and smaller (light blue) activities for the SE/ EF and SF/EE, respectively. For the ipsilateral-only loads (red traces), the same pattern emerges, except in the opposite direction with larger (light red) and smaller (dark red) activities for the SF/EE and SE/EF, respectively. As a result, during the mirror context, the resulting activities cancel out (Fig. 9I, middle); whereas for the opposite context, the activities sum to generate substantial activity (Fig. 9I, right). However, the size of the opposite activity is smaller than predicted by the additive model. For the top load-dependent components, the reverse happens as these components captured greater variance for the mirror context and additive mirror model (Fig. 9C, components 3 and 4; Fig. $9 D$, components 3 and 5) than the opposite context and additive opposite model (Fig. 9E,F). Examining the dynamics in the load-dependent components revealed the contralateral and ipsilateral-related activities summed to generate substantial activity during the mirror context and cancelled out during the opposite context (Fig. 9J). Last, the top time component captured similar amounts of variance for the unimanual and bimanual contexts (Fig. 9A,C,E, component 5; Fig. 9B,D,F, component 4). Examining the dynamics in the time component revealed similar activities for both the unimanual (Fig. 9K, left) and bimanual contexts (Fig. $9 K$, middle and right); however, the bimanual activities were smaller than the additive models. These results highlight that limb-dependent and limb-independent components of M1 activity largely transfer across the unimanual and bimanual contexts similar to an additive model. However, bimanual activity appeared at times to be smaller than predicted from the additive model.

Next, we explored whether this subadditivity was specific to the representation of a particular limb. For this, we exploited the fact that ipsilateral- and contralateral-related activities in M1 could be isolated into orthogonal subspaces during unimanual movements (Ames and Churchland, 2019; Heming et al., 2019). We identified the unique subspaces for the ipsilateral- and contralateral-related activities using a joint optimization technique (Elsayed et al., 2016; Heming et al., 2019). This optimizer finds components that capture the largest amount of variance for the ipsilateral-only and contralateral-only activities while constraining the components to be orthogonal. Figure 10A, E shows the variance accounted for (VAF) by the 10 dimensions that span 


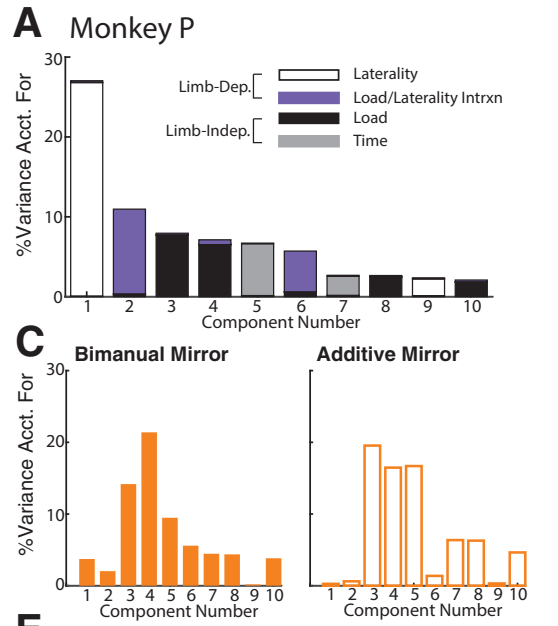

E

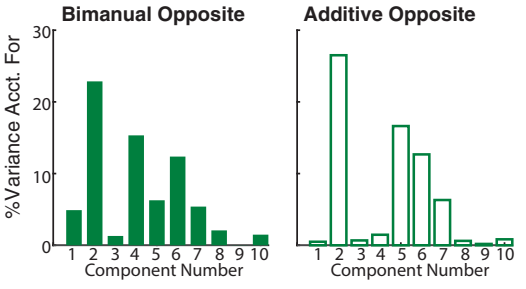

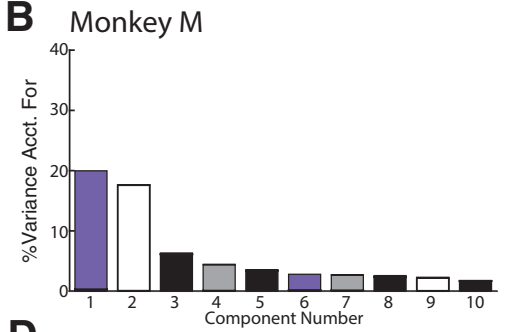

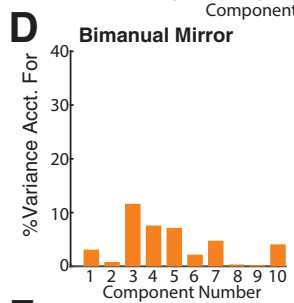

Additive Mirror
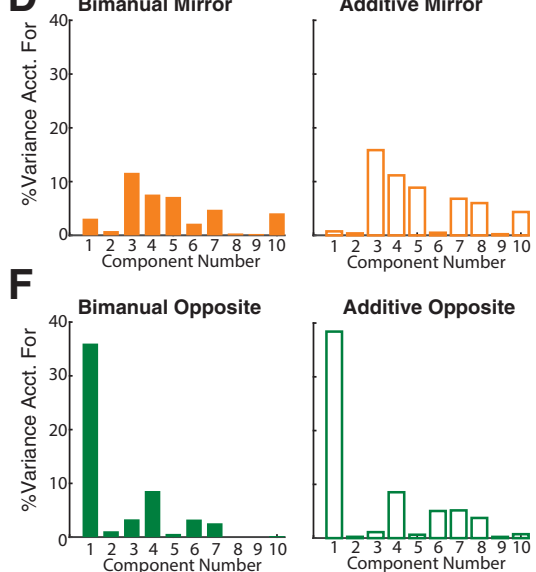

Additive Opposite

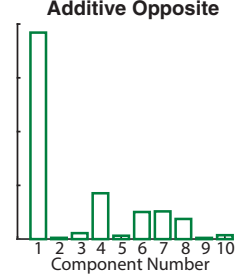

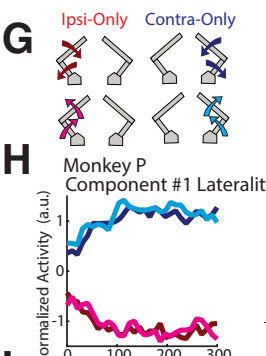

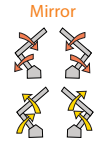

Opposite

息

站

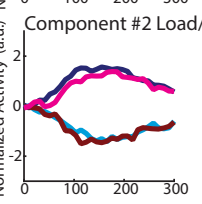

J 高

F. Component \#3 Load

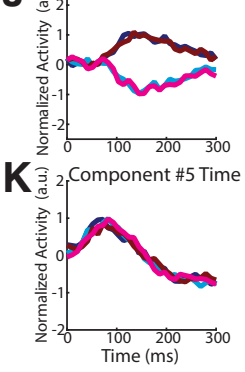

- - - Bimanual

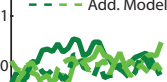

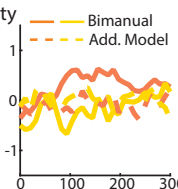
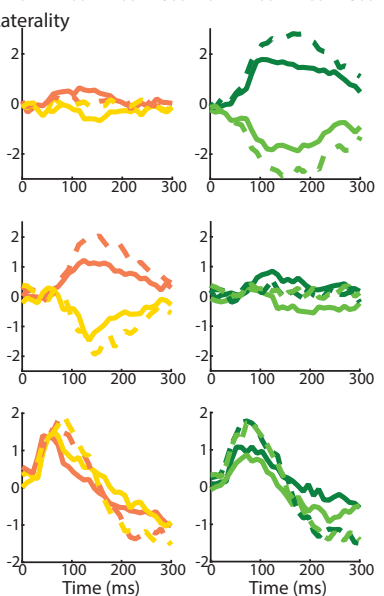
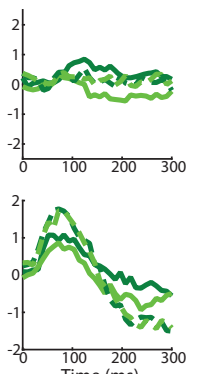

Figure 9. dPCA was applied to the contralateral-only and ipsilateral-only activities to identify activity patterns that were limb-dependent (laterality and load/laterality interaction) and limbindependent (load and time). $\boldsymbol{A}$, Components identified using APCA ordered based on the amount of variance captured for Monkey P. Bar colors represent the amount of variance captured by a particular task parameter. For example, component 1 captures a substantial amount of the laterality-related activity (open bar) and very little of the variance for the other task parameters (purple, black, and gray bars). $\boldsymbol{B}$, Same as in $\boldsymbol{A}$, but for Monkey M. C, The variance accounted for the mirror activity (left) and additive mirror model (right) by each component identified in $\boldsymbol{A}$. $\boldsymbol{D}$, Same as in $\boldsymbol{C}$, but for Monkey M. $\boldsymbol{E}, \boldsymbol{F}$, Same as in $\boldsymbol{C}, \boldsymbol{D}$, but for the opposite activity and additive opposite model. $\boldsymbol{G}$, Diagrams represent the applied loads and their associated colors. $\boldsymbol{H}$, The dynamics that reside in component 1 for Monkey $P$, which captures largely laterality-related activity. Left, Dynamics for the ipsilateral-only and contralateral-only loads. Middle, Dynamics for the mirror context (solid) and the additive mirror model (dashed). Right, Dynamics for the opposite context (solid) and additive opposite model (dashed). I, Same as in $\boldsymbol{H}$, but for component 2 , which captures activity reflecting the laterality/load interactions. J, Same as in $\boldsymbol{H}$, but for component 3, which captures load-related activity. $\boldsymbol{K}$, Same as in $\boldsymbol{H}$, but for component 5, which captures time-only activity.

the ipsilateral subspaces for Monkeys $\mathrm{P}$ and $\mathrm{M}$, respectively. This subspace captured a substantial amount of the ipsilateral-only variance (Monkey P/Monkey M: 75\%/60\%) and little of the contralateral-only variance $(5 \% / 8 \%)$. This subspace also captured a substantial amount of the variance for the mirror $(22 \% / 21 \%)$ and opposite $(16 \% / 21 \%)$ contexts; however, it was noticeably smaller than the amount captured for the additive mirror $(31 \% /$ $28 \%$ ) and opposite models (34\%/30\%).

Figure $10 C, G$ shows the VAF by the 10 dimensions that span the contralateral subspace for Monkeys $\mathrm{P}$ and $\mathrm{M}$, respectively. For Monkey $\mathrm{P} /$ Monkey $\mathrm{M}$, this subspace captured a substantial amount of the contralateral-only variance $(80 \% / 69 \%)$ and little of the ipsilateral-only variance (5\%/9\%). This subspace also captured a substantial amount of the variance for the mirror (53\%/ $29 \%$ ) and opposite (60\%/45\%) contexts and was comparable to the amount of variance captured for the additive mirror (52\%/ $42 \%)$ and opposite (49\%/48\%) models.

We quantified how well the ipsilateral and contralateral subspaces aligned with the mirror and opposite subspaces by calculating an alignment index. The alignment index can range from 0 (indicating the subspaces were orthogonal with respect to each other) to 1 (indicating complete alignment between the subspaces). However, we have demonstrated previously how ipsilateraland contralateral-related activities reside in orthogonal subspace and have an alignment index near 0 (Heming et al., 2019). Thus, comparing the ipsilateral subspace with the bimanual subspace will result in a small alignment index simply because of the contralateral-related activity. We mitigated this problem by first projecting the bimanual activity into the null space of the contralateral components (see Materials and Methods). A similar procedure was also used when calculating the contralateral alignment index. For comparison, we generated a null distribution that compared the alignment of randomly sampled subspaces. For both monkeys, the alignment indices for the ipsilateral components resided near 0.55 (Fig. 10B,F; Monkey P/Monkey M: mirror $=0.62 / 0.47$; opposite $=0.51 / 0.60)$ and were lower than the additive model $($ mirror $=0.93 / 0.84$; opposite $=0.93 / 0.91)$. The indices also resided within or near the random distribution (mirror $p=0.17 / p=0.4$, opposite $p=0.83 / p=0.007$ ). In contrast, the alignment indices for the contralateral components resided near 0.75 (Fig. $10 D, H$; mirror $=0.81 / 0.57$, opposite $=$ $0.82 / 0.78$ ) and were significantly greater than the random distribution (mirror $=p<0.001 / p=0.02$, opposite $p<0.001 / p<$ $0.001)$; however, they were still lower than the additive models (mirror $=0.96 / 0.92$, opposite $=0.98 / 0.95)$. These results suggest that activity was better maintained in the contralateral subspace than the ipsilateral subspace.

\section{Linear versus nonlinear}

Several studies have suggested that representations for the contralateral and ipsilateral limbs are nonlinearly combined during bimanual control (Yokoi et al., 2011; Diedrichsen et al., 2013). We investigated whether nonlinear effects were present in our data by comparing a model with linear terms for the contralateral and ipsilateral loads (linear model) with a model that included linear and nonlinear interaction terms for the contralateral and 

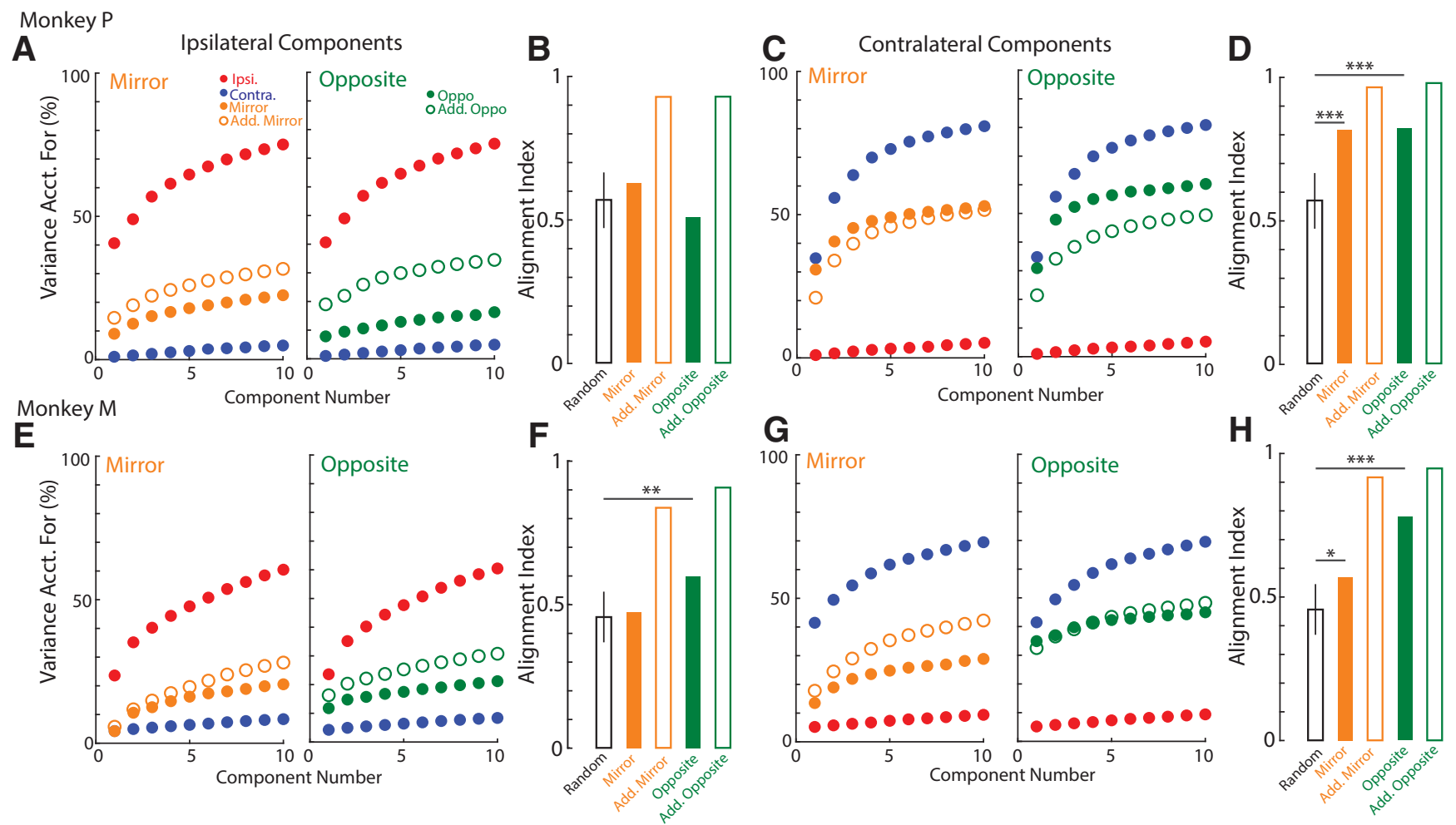

Figure 10. Subspace analysis. $A$, Left, The variance accounted for by the ipsilateral subspace for the contralateral-only (blue), ipsilateral-only (red), mirror (yellow solid), and additive mirror model (yellow open). Right. The same as left, but for the opposite (green solid) and additive opposite model (green open). The contralateral-only and ipsilateral-only activities are the same in the left and right panels. Data are plotted as a cumulative sum over the subspace components. $\boldsymbol{B}$, Alignment indices were calculated between the top 5 ipsilateral components and the activities for the mirror, opposite and additive models. Random reflects randomly sampling from the data covariance matrix. $\boldsymbol{C}, \boldsymbol{D}$, Same as in $\boldsymbol{A}, \boldsymbol{B}$, but for the contralateral subspace. $\boldsymbol{E}-\boldsymbol{H}$, Same as in $A-D$, but for Monkey M. ${ }^{*} p<0.05,{ }^{* *} p<0.01,{ }^{* * *} p<0.001$.

ipsilateral loads (nonlinear model). Figure $11 A, E$ compares the VAF by the linear (abscissa) and nonlinear models (ordinate) during the perturbation epoch for Monkeys $\mathrm{P}$ and $\mathrm{M}$, respectively. We found that the linear model captured $89 \% / 74 \%$ of the variance for Monkey P/Monkey M, whereas the nonlinear model captured $93 \% / 89 \%$ of variance. Also, we found all neurons resided above the unity line consistent with the fact that the nonlinear model had twice as many free parameters. We assessed model performance using AIC, which balances how well a model fits with the number of free parameters. Models with lower AIC are preferred over models with larger AIC. Figure $11 B, F$ shows the differences between the AIC for the linear and nonlinear models as a cumulative sum for Monkeys $\mathrm{P}$ and $\mathrm{M}$, respectively. The cumulative sums reside to the left of the zero-line, indicating that $97 \%$ and $81 \%$ of neurons were better described (i.e., lower AIC) for the linear model than the nonlinear model for Monkeys $\mathrm{P}$ and $\mathrm{M}$, respectively. Examining the steady state, we also found that the nonlinear model accounted for $6 \% / 9 \%$ more variance than the linear model for Monkey P/Monkey M (Fig. 11C,G). However, all neurons were better described by the linear model (Fig. 11D,H).

\section{Control analysis for overlapping neurons}

We pooled neurons across multiple recording sessions spaced out by $>3$ months and treated each neuron as independent samples. However, recently Gallego et al. (2020) suggested that some neurons may be recorded on chronic arrays for more than 1 year. Thus, our assumption of independently sampled neurons may not be valid and could affect our results. We mitigated this effect by verifying our major findings using a nonoverlapping subset of the neuron population (see Materials and Methods) composed of 58 and 93 neurons for Monkeys $\mathrm{P}$ and $\mathrm{M}$, respectively. We found for Monkey P/Monkey M that 25/42 neurons responded to the unimanual and bimanual loads during the perturbation epoch and 21/40 neurons responded unimanual and bimanual loads during the steady-state epoch. We found a suppression of the ipsilateral-related activity during the bimanual loads compared with the ipsilateral-only loads for the perturbation (Monkey P/Monkey M: relative size of bimanual to unimanual: $70 \% / 75 \%$, paired $t$ test: $t_{(24)}=3.5 / t_{(41)}=4.1, p=0.002 / p<$ $0.001)$ and steady-state epochs $\left(78 \% / 81 \%\right.$, paired $t$ test: $t_{(20)}=$ $\left.2.5 / t_{(39)}=2.9, p=0.02 / p=0.01\right)$. Similar results were found for the contralateral-related activity during the perturbation $(90 \% /$ $78 \%$, paired $t$ test: $\left.t_{(24)}=2.1 / t_{(41)}=5.3, p=0.04 / p<0.001\right)$ and steady-state epochs $\left(63 \% / 84 \%\right.$, paired $t$ test: $t_{(20)}=4.8 / t_{(39)}=3.7$, $p<0.001 / p<0.001)$. We also found that neurons largely maintained their tuning for the ipsilateral loads across the unimanual and bimanual loads for the perturbation $(\mathrm{R}=0.69 / 0.63$, for uniform and within-context $p<0.001 / p<0.001)$ and steady-state epochs $(\mathrm{R}=0.66 / 0.76$, for uniform $p<0.001 / p<0.001$, withincontext $p<0.001 / p=0.16)$. However, we found that neurons better maintained their tuning for the contralateral loads across the unimanual and bimanual loads for the perturbation $(\mathrm{R}=$ $0.82 / 0.83$, for uniform and within-context: $p<0.001 / p<0.001)$ and steady-state epochs $(\mathrm{R}=0.91 / 0.81$, uniform: $p<0.001 /$ $p<0.001$; within-context: $p=0.01 / p<0.001)$. Lastly, we found that the ipsilateral components had an alignment index with the bimanual activity near 0.6 (mirror $=0.69 / 0.49$, additive mirror $0.92 / 0.84, p=0.05 / p=0.12$, opposite $=0.6 / 0.59$, additive opposite $=$ $0.92 / 0.89, p=0.4 / p<0.01$ ), whereas the contralateral components had an alignment index with the bimanual activity near 0.78 ( mirror $=0.86 / 0.58$, additive mirror $0.97 / 0.9$, opposite $=0.88 / 0.78$, 
A

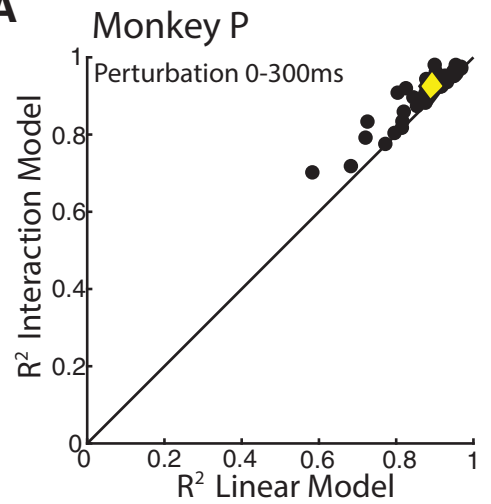

E

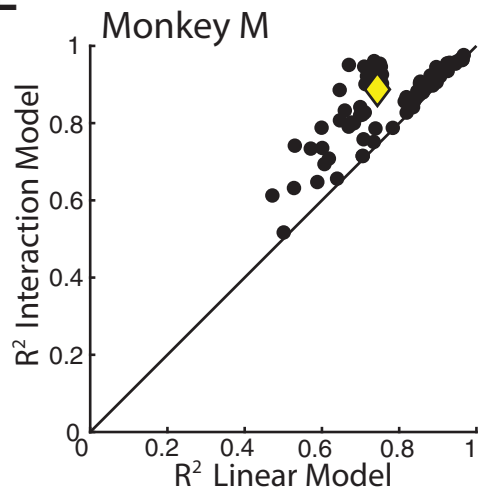

B

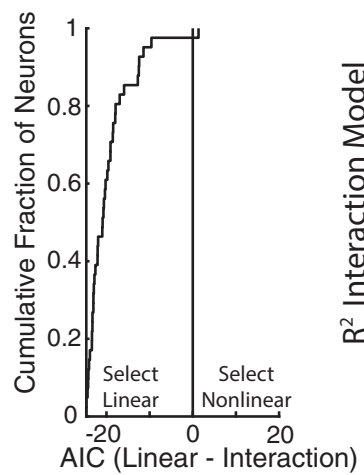

$\mathbf{F}$

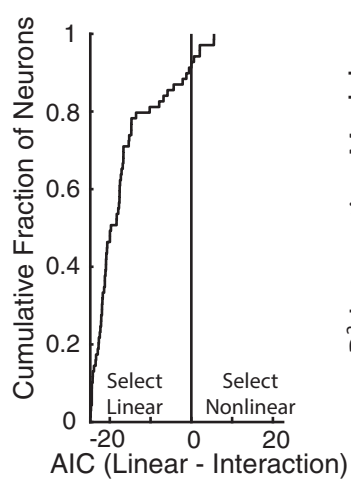

C

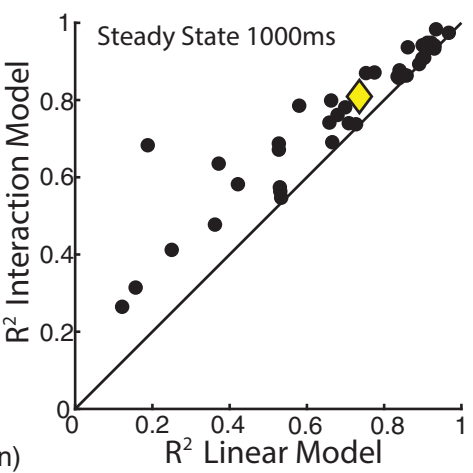

G

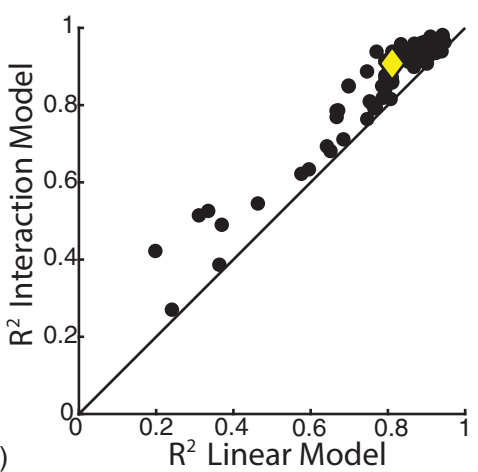

D

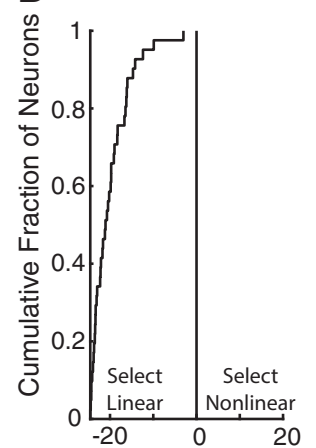

AIC (Linear - Interaction)

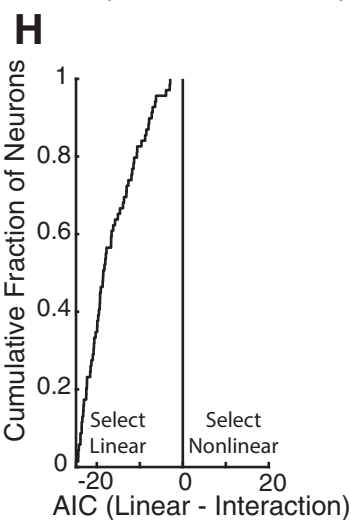

Figure 11. Comparison of the linear and nonlinear models. $\boldsymbol{A}$, Comparison of model fits between the linear and nonlinear models for each neuron. Yellow diamond represents the median. $\boldsymbol{B}$, Difference between the AICs calculated for the linear and nonlinear models. Differences that are $<0$ indicate the linear model should be selected, whereas differences $>0$ indicate the nonlinear model should be selected. $\boldsymbol{C}, \boldsymbol{D}$, Same as in $\boldsymbol{A}, \boldsymbol{B}$, but for the steady-state epoch. $\boldsymbol{E}-\boldsymbol{H}$, Same as in $\boldsymbol{A}-\boldsymbol{D}$, but for Monkey $\mathbf{M}$.

additive opposite $=0.98 / 0.93, p<0.01$ for all comparisons). Thus, we found essentially the same results among the nonoverlapping neuron population.

\section{Discussion}

We investigated whether limb representations in M1 change between unimanual and bimanual motor actions. We found a substantial overlap ( $83 \%$ ) between neurons that were responsive to loads applied to either arm (unimanual) and to loads applied to both arms simultaneously (bimanual). There was a small reduction $(\sim 25 \%)$ of activity related to each limb during the bimanual task. Neurons also maintained similar preferred loads with a greater change in tuning for the ipsilateral-related activity (change in unimodal distribution from the additive model of $\sim 25 \%$ ) than the contralateral-related activity ( $~ 9 \%)$. Lastly, we found that the subspace identified for the unimanual loads captured a substantial amount of the variance for the bimanual loads. These data highlight how M1 largely maintains its representations of the ipsilateral and contralateral limbs during bimanual control.

Studies demonstrate that M1's representation of the contralateral limb remains stable across time for a given behavior (Scott and Kalaska, 1997; Chestek et al., 2007; Stevenson et al., 2011). M1 also maintains this representation when adapting to a novel environment (Cherian et al., 2013; Perich and Miller, 2017; Perich et al., 2018; Vyas et al., 2018) and when performing various forms of reaching (Gribble and Scott, 2002; Yakovenko and Drew, 2015; Gallego et al., 2018; Lara et al., 2018). In contrast, large changes in the neural representation have been observed across behavioral tasks (Cheney and Fetz, 1980; Muir and Lemon, 1983; Drew et al., 1996). For example, M1 activity during reaching and locomotion reflects distinct subspaces (Miri et al., 2017). Furthermore, load representations can change dramatically across postural control and reaching, although neurons still maintain similar tuning for the loads (Kurtzer et al., 2005; Heming et al., 2016). Thus, M1's representation remains relatively constant for a given behavior but can show substantial changes across behaviors.

Here, we found that the contralateral representation remained stable across unimanual and bimanual contexts. There was a reduction in activity that may reflect a corresponding reduction in the motor output. We cannot rule this out as we did not record muscle activity, but hand kinematics were similar between unimanual and bimanual loads for the first $300 \mathrm{~ms}$ after the load was applied. Furthermore, we observed a similar reduction during the steady-state epoch when motor output should be comparable between the unimanual and bimanual loads. Importantly, the preferred load directions remained quite constant, and the subspace identified during the unimanual task captured a substantial amount of variance during the bimanual context. Thus, there was a small reduction in activity, but the basic pattern of activity across behavioral contexts remained stable. Similar results were found for the ipsilateral representation, although the ipsilateral tuning changed more and the subspace captured less variance during the bimanual loads. Thus, while a substantive proportion of the ipsilateral representation was maintained, it was less than that observed for the contralateral limb. The ability to simultaneously represent both limbs while performing bimanual motor actions may reflect that the subspaces associated with each limb were orthogonal.

In contrast, premotor cortical regions show a greater change in neural representations between unimanual and bimanual 
motor actions (Tanji et al., 1987, 1988; Rokni et al., 2003; Willett et al., 2020). During bimanual movements, Willett et al. (2020) found relatively small reductions in the contralateral representation in the premotor cortex of humans but found $\sim 50 \%$ reductions for the ipsilateral representation. Interestingly, they found that the ipsilateral and contralateral representations were in subspaces that overlapped more than M1's representations. Cisek et al. (2003) also found that the preferred directions of premotor neurons during reaching were correlated for the two limbs. This lack of orthogonality in premotor regions may result in a reduction of the ipsilateral representation to reduce interference during bimanual motor actions (Rokni et al., 2003; Willett et al., 2020).

Although speculative, these differences in the organization of ipsilateral and contralateral representations may reflect the types of information that are represented in these cortical areas. Studies highlight that premotor cortical activity is more related to extrinsic features of motor actions, whereas M1 activity is more related to intrinsic features related to the motor periphery (Evarts, 1968; Humphrey, 1972; Cheney and Fetz, 1980; Fromm, 1983; Werner et al., 1991; Scott and Kalaska, 1997; Scott et al., 1997; Shen and Alexander, 1997a,b). It may be that goal-related features of a task are more broadly expressed across the entire premotor network. In natural situations, this broad expression of the behavioral goal may prove valuable to permit rapid alternate motor strategies to attain the goal, such as using the other limb to reach for an object. In contrast, when there are independent goals for different motor effectors the premotor representation of the goal associated with the appropriate effector is maintained while the other goal representations are suppressed. In contrast, M1 activity is more related to the motor execution, which is more effector specific and resides closer to downstream motor targets. Thus, M1 exhibits independent representations of the two limbs, but this allows both representations to be maintained during bimanual motor actions.

Previous studies by Vaadia and colleagues had explored bimanual coordination in M1 (Steinberg et al., 2002; Rokni et al., 2003). However, their population of neurons exhibited functional properties more similar to premotor cortex. They found neurons had similar tuning for the contralateral and ipsilateral limbs during unimanual reaches (Steinberg et al., 2002). They also found a substantial change in a neuron's preferred direction and an $\sim 50 \%$ reduction in magnitude for the ipsilateral-related activity between unimanual and bimanual reaches (Rokni et al., 2003). This may reflect some fluidity in ipsilateral representations across animals or behavioral tasks, postural versus reaching. Alternatively, their M1 recordings may be from the transition zone between premotor cortex and M1, which exhibits properties reflecting a mixture of the two areas (Cisek et al., 2003).

We used floating microelectrode arrays to record from M1 that was positioned on the surface of the precentral gyrus. As a result, we did not sample from the most caudal portion of M1, which lies in the bank of the central sulcus. Studies have suggested a rostrocaudal gradient across motor cortex for several attributes. Caudal motor cortex exhibits a greater number of corticomotor neurons (Rathelot and Strick, 2009; Witham et al., 2016), greater independence of tuning between the upper limbs (Cisek et al., 2003), decreased preparatory activity (Crammond and Kalaska, 2000), and greater steady-state activity during postural control (Crammond and Kalaska, 1996) than rostral motor cortex (i.e., premotor cortex). If a gradient does exist, then caudal M1 likely also maintains orthogonal subspaces for the ipsilateral and contralateral limbs but may show even less reduction in activity during bimanual motor tasks than rostral M1.

Parietal reach region also displays neural representations related to motor actions of both limbs (Kermadi et al., 2000; Chang et al., 2008; Mooshagian et al., 2018). Parietal reach region is primarily involved with controlling the contralateral limb (Chang et al., 2008; Yttri et al., 2013); however, neurons in parietal reach region respond before movements of the contralateral and ipsilateral limbs as well as upcoming saccades (Chang et al., 2008; Chang and Snyder, 2012). However, this ipsilateral activity is predominantly related to a sensory response to the visual target, whereas the contralateral activity is related to the sensory event and motor planning (Mooshagian et al., 2018). During bimanual actions, parietal reach region also suppresses the ipsilateral-related activity similar to premotor cortex (Mooshagian et al., 2018).

It is not clear whether representing both limbs by one hemisphere and the change to these representations during bimanual motor actions influences actual motor function. Given the behavioral goal was identical for a given limb during unimanual and bimanual tasks, one might expect that any change in the neural representations might impact control. As stated above, we did not observe substantive changes in the kinematics of movement in this relatively simple postural perturbation task. However, the motor system appears to prefer mirror symmetric movements of the limb even when instructed to perform antisymmetric movements (Kelso, 1984). Furthermore, learning a force field while performing a unimanual reach only partially transfers to the equivalent bimanual reach (Nozaki et al., 2006; Nozaki and Scott, 2009; Howard et al., 2010). These observations may reflect interactions between the ipsilateral and contralateral representations in motor cortex during bimanual motor tasks.

The bimanual representations in motor cortex may support behaviors where the two limbs are coordinated together to perform a common goal. Currently, most neurophysiological investigations of bimanual control, including our own, have used tasks where the goals for each limb are independent, thus requiring minimal interlimb coordination (Donchin et al., 1998; Steinberg et al., 2002; Rokni et al., 2003; Willett et al., 2020). Future studies should investigate behaviors that require interlimb coordination to attain a common goal (Diedrichsen, 2007; Dimitriou et al., 2012; Córdova Bulens et al., 2017). In these contexts, sensory feedback from one limb can elicit goal-directed motor actions in the opposite limb in $\sim 70 \mathrm{~ms}$ (Diedrichsen, 2007; Mutha and Sainburg, 2009; Omrani et al., 2013). It is likely that these interlimb feedback responses involve interactions between these overlapping subspaces in motor cortex.

\section{References}

Ames KC, Churchland MM (2019) Motor cortex signals for each arm are mixed across hemispheres and neurons yet partitioned within the population response. eLife 8:e46159.

Batschelet E (1981) Circular statistics in biology. San Diego: Academic.

Berlot E, Prichard G, O’Reilly J, Ejaz N, Diedrichsen J (2019) Ipsilateral finger representations in the sensorimotor cortex are driven by active movement processes, not passive sensory input. J Neurophysiol 121:418-426.

Brösamle C, Schwab ME (1997) Cells of origin, course, and termination patterns of the ventral, uncrossed component of the mature rat corticospinal tract. J Comp Neurol 386:293-303.

Burnham KP, Anderson DR (2004) Model selection and multimodel inference: a practical information-theoretic approach, Ed 2. New York: Springer.

Cabel DW, Cisek P, Scott SH (2001) Neural activity in primary motor cortex related to mechanical loads applied to the shoulder and elbow during a postural task. J Neurophysiol 86:2102-2108. 
Chang SW, Snyder LH (2012) The representations of reach endpoints in posterior parietal cortex depend on which hand does the reaching. J Neurophysiol 107:2352-2365.

Chang SW, Dickinson AR, Snyder LH (2008) Limb-specific representation for reaching in the posterior parietal cortex. J Neurosci 28:6128-6140.

Cheney PD, Fetz EE (1980) Functional classes of primate corticomotoneuronal cells and their relation to active force. J Neurophysiol 44:773-791.

Cherian A, Fernandes HL, Miller LE (2013) Primary motor cortical discharge during force field adaptation reflects muscle-like dynamics. J Neurophysiol 110:768-783.

Chestek CA, Batista AP, Santhanam G, Yu BM, Afshar A, Cunningham JP, Gilja V, Ryu SI, Churchland MM, Shenoy KV (2007) Single-neuron stability during repeated reaching in macaque premotor cortex. J Neurosci 27:10742-10750.

Cisek P, Crammond DJ, Kalaska JF (2003) Neural activity in primary motor and dorsal premotor cortex in reaching tasks with the contralateral versus ipsilateral arm. J Neurophysiol 89:922-942.

Córdova Bulens D, Crevecoeur F, Thonnard JL, Lefèvre P (2017) Optimal use of limb mechanics distributes control during bimanual tasks. J Neurophysiol 119:921-932.

Cramer SC, Finklestein SP, Schaechter JD, Bush G, Rosen BR (1999) Activation of distinct motor cortex regions during ipsilateral and contralateral finger movements. J Neurophysiol 81:383-387.

Crammond DJ, Kalaska JF (1996) Differential relation of discharge in primary motor cortex and premotor cortex to movements versus actively maintained postures during a reaching task. Exp Brain Res 108:45-61.

Crammond DJ, Kalaska JF (2000) Prior information in motor and premotor cortex: activity during the delay period and effect on pre-movement activity. J Neurophysiol 84:986-1005.

Diedrichsen J (2007) Optimal task-dependent changes of bimanual feedback control and adaptation. Curr Biol 17:1675-1679.

Diedrichsen J, Wiestler T, Krakauer JW (2013) Two distinct ipsilateral cortical representations for individuated finger movements. Cereb Cortex 23:1362-1377.

Dimitriou M, Franklin DW, Wolpert DM (2012) Task-dependent coordination of rapid bimanual motor responses. J Neurophysiol 107:890-901.

Donchin O, Gribova A, Steinberg O, Bergman H, Vaadia E (1998) Primary motor cortex is involved in bimanual coordination. Nature 395:274-278.

Downey JE, Quick KM, Schwed N, Weiss JM, Wittenberg GF, Boninger ML, Collinger JL (2020) The motor cortex has independent representations for ipsilateral and contralateral arm movements but correlated representations for grasping. Cereb Cortex Advance online publication. Retrieved June 4, 2020. doi: 10.1093/cercor/bhaa120.

Drew T, Jiang W, Kably B, Lavoie S (1996) Role of the motor cortex in the control of visually triggered gait modifications. Can J Physiol Pharmacol 74:426-442.

Dum RP, Strick PL (1996) Spinal cord terminations of the medial wall motor areas in macaque monkeys. J Neurosci 16:6513-6525.

Elsayed GF, Lara AH, Kaufman MT, Churchland MM, Cunningham JP (2016) Reorganization between preparatory and movement population responses in motor cortex. Nat Commun 7:13239.

Evarts EV (1968) Relation of pyramidal tract activity to force exerted during voluntary movement. J Neurophysiol 31:14-27.

Fromm C (1983) Changes of steady state activity in motor cortex consistent with the length-tension relation of muscle. Pflugers Arch 398:318-323.

Gallego JA, Perich MG, Chowdhury RH, Solla SA, Miller LE (2020) Longterm stability of cortical population dynamics underlying consistent behavior. Nat Neurosci 23:260-270.

Gallego JA, Perich MG, Naufel SN, Ethier C, Solla SA, Miller LE (2018) Cortical population activity within a preserved neural manifold underlies multiple motor behaviors. Nat Commun 9:4233.

Ganguly K, Secundo L, Ranade G, Orsborn A, Chang EF, Dimitrov DF, Wallis JD, Barbaro NM, Knight RT, Carmena JM (2009) Cortical representation of ipsilateral arm movements in monkey and man. J Neurosci 29:12948-12956.

Gribble PL, Scott SH (2002) Overlap of internal models in motor cortex for mechanical loads during reaching. Nature 417:938-941.

Heming EA, Lillicrap TP, Omrani M, Herter TM, Pruszynski JA, Scott SH (2016) Primary motor cortex neurons classified in a postural task predict muscle activation patterns in a reaching task. J Neurophysiol 115:20212032.
Heming EA, Cross KP, Takei T, Cook DJ, Scott SH (2019) Independent representations of ipsilateral and contralateral limbs in primary motor cortex. eLife 8:e48190.

Herter TM, Korbel T, Scott SH (2009) Comparison of neural responses in primary motor cortex to transient and continuous loads during posture. J Neurophysiol 101:150-163.

Howard IS, Ingram JN, Wolpert DM (2010) Context-dependent partitioning of motor learning in bimanual movements. J Neurophysiol 104:20822091.

Humphrey DR (1972) Relating motor cortex spike trains to measures of motor performance. Brain Res 40:7-18.

Kaufman MT, Seely JS, Sussillo D, Ryu SI, Shenoy KV, Churchland MM (2016) The largest response component in the motor cortex reflects movement timing but not movement type. eNeuro 3:ENEURO.008516.2016

Kelso JA (1984) Phase transitions and critical behavior in human bimanual coordination. Am J Physiol 246:R1000-R1004

Kermadi I, Liu Y, Tempini A, Calciati E, Rouiller EM (1998) Neuronal activity in the primate supplementary motor area and the primary motor cortex in relation to spatio-temporal bimanual coordination. Somatosens Mot Res 15:287-308.

Kermadi I, Liu Y, Rouiller EM (2000) Do bimanual motor actions involve the dorsal premotor (PMd), cingulate (CMA) and posterior parietal (PPC) cortices? Comparison with primary and supplementary motor cortical areas. Somatosens Mot Res 17:255-271.

Kobak D, Brendel W, Constantinidis C, Feierstein CE, Kepecs A, Mainen ZF, Qi XL, Romo R, Uchida N, Machens CK (2016) Demixed principal component analysis of neural population data. eLife 5:e10989.

Kurtzer I, Herter TM, Scott SH (2005) Random change in cortical load representation suggests distinct control of posture and movement. Nat Neurosci 8:498-504.

Kuypers HG (2011) Anatomy of the descending pathways. In: Comprehensive physiology, pp 597-666. Atlanta: American Cancer Society.

Lacroix S, Havton LA, McKay H, Yang H, Brant A, Roberts J, Tuszynski MH (2004) Bilateral corticospinal projections arise from each motor cortex in the macaque monkey: a quantitative study. J Comp Neurol 473:147-161.

Lara AH, Elsayed GF, Zimnik AJ, Cunningham JP, Churchland MM (2018) Conservation of preparatory neural events in monkey motor cortex regardless of how movement is initiated. eLife 7:e31826.

Miri A, Warriner CL, Seely JS, Elsayed GF, Cunningham JP, Churchland MM, Jessell TM (2017) Behaviorally selective engagement of short-latency effector pathways by motor cortex. Neuron 95:683-696.e11.

Montgomery LR, Herbert WJ, Buford JA (2013) Recruitment of ipsilateral and contralateral upper limb muscles following stimulation of the cortical motor areas in the monkey. Exp Brain Res 230:153-164.

Mooshagian E, Wang C, Holmes CD, Snyder LH (2018) Single units in the posterior parietal cortex encode patterns of bimanual coordination. Cereb Cortex 28:1549-1567.

Muir RB, Lemon RN (1983) Corticospinal neurons with a special role in precision grip. Brain Res 261:312-316.

Mutha PK, Sainburg RL (2009) Shared bimanual tasks elicit bimanual reflexes during movement. J Neurophysiol 102:3142-3155.

Nozaki D, Scott SH (2009) Multi-compartment model can explain partial transfer of learning within the same limb between unimanual and bimanual reaching. Exp Brain Res 194:451-463.

Nozaki D, Kurtzer I, Scott SH (2006) Limited transfer of learning between unimanual and bimanual skills within the same limb. Nat Neurosci 9:1364-1366.

Omrani M, Diedrichsen J, Scott SH (2013) Rapid feedback corrections during a bimanual postural task. J Neurophysiol 109:147-161.

Perich MG, Miller LE (2017) Altered tuning in primary motor cortex does not account for behavioral adaptation during force field learning. Exp Brain Res 235:2689-2704.

Perich MG, Gallego JA, Miller L (2018) A neural population mechanism for rapid learning. Neuron 100:964-976.

Pruszynski JA, Omrani M, Scott SH (2014) Goal-dependent modulation of fast feedback responses in primary motor cortex. J Neurosci 34:46084617.

Rathelot JA, Strick PL (2009) Subdivisions of primary motor cortex based on cortico-motoneuronal cells. Proc Natl Acad Sci USA 106:918-923.

Rokni U, Steinberg O, Vaadia E, Sompolinsky H (2003) Cortical representation of bimanual movements. J Neurosci 23:11577-11586. 
Rosenzweig ES, Brock JH, Culbertson MD, Lu P, Moseanko R, Edgerton VR, Havton LA, Tuszynski MH (2009) Extensive spinal decussation and bilateral termination of cervical corticospinal projections in rhesus monkeys. J Comp Neurol 513:151-163.

Rouse AG, Schieber MH (2018) Condition-dependent neural dimensions progressively shift during reach to grasp. Cell Rep 25:3158-3168.e3.

Scott SH (1999) Apparatus for measuring and perturbing shoulder and elbow joint positions and torques during reaching. J Neurosci Methods 89:119127

Scott SH, Kalaska JF (1997) Reaching movements with similar hand paths but different arm orientations: I. Activity of individual cells in motor cortex. J Neurophysiol 77:826-852.

Scott SH, Sergio LE, Kalaska JF (1997) Reaching movements with similar hand paths but different arm orientations: II. Activity of individual cells in dorsal premotor cortex and parietal area 5. J Neurophysiol 78:24132426.

Shen L, Alexander GE (1997a) Preferential representation of instructed target location versus limb trajectory in dorsal premotor area. J Neurophysiol 77:1195-1212.

Shen L, Alexander GE (1997b) Neural correlates of a spatial sensory-tomotor transformation in primary motor cortex. J Neurophysiol 77:11711194.

Soteropoulos DS, Edgley SA, Baker SN (2011) Lack of evidence for direct corticospinal contributions to control of the ipsilateral forelimb in monkey. J Neurosci 31:11208-11219.

Steinberg O, Donchin O, Gribova A, De Oliveira SC, Bergman H, Vaadia E (2002) Neuronal populations in primary motor cortex encode bimanual arm movements: population vectors in bimanual movements. Eur J Neurosci 15:1371-1380.

Stevenson IH, Cherian A, London BM, Sachs NA, Lindberg E, Reimer J, Slutzky MW, Hatsopoulos NG, Miller LE, Kording KP (2011) Statistical assessment of the stability of neural movement representations. J Neurophysiol 106:764-774.
Takei T, Crevecoeur F, Herter TM, Cross KP, Scott SH (2018) Correlations between primary motor cortex activity with recent past and future limb motion during unperturbed reaching. J Neurosci 38:7787-7799.

Tanji J, Okano K, Sato KC (1987) Relation of neurons in the nonprimary motor cortex to bilateral hand movement. Nature 327:618-620.

Tanji J, Okano K, Sato KC (1988) Neuronal activity in cortical motor areas related to ipsilateral, contralateral, and bilateral digit movements of the monkey. J Neurophysiol 60:325-343

Thompson KG, Hanes DP, Bichot NP, Schall JD (1996) Perceptual and motor processing stages identified in the activity of macaque frontal eye field neurons during visual search. J Neurophysiol 76:4040-4055.

Trautmann EM, Stavisky SD, Lahiri S, Ames KC, Kaufman MT, O’Shea DJ, Vyas S, Sun X, Ryu SI, Ganguli S, Shenoy KV (2019) Accurate estimation of neural population dynamics without spike sorting. Neuron 103:292-308.e4.

Vyas S, Even-Chen N, Stavisky SD, Ryu SI, Nuyujukian P, Shenoy KV (2018) Neural population dynamics underlying motor learning transfer. Neuron 97:1177-1186.e3.

Werner W, Bauswein E, Fromm C (1991) Static firing rates of premotor and primary motor cortical neurons associated with torque and joint position. Exp Brain Res 86:293-302.

Willett FR, Deo DR, Avansino DT, Rezaii P, Hochberg LR, Henderson JM, Shenoy KV (2020) Hand knob area of premotor cortex represents the whole body in a compositional way. Cell 181:396-409.e26.

Witham CL, Fisher KM, Edgley SA, Baker SN (2016) Corticospinal inputs to primate motoneurons innervating the forelimb from two divisions of primary motor cortex and area 3a. J Neurosci 36:2605-2616.

Yakovenko S, Drew T (2015) Similar motor cortical control mechanisms for precise limb control during reaching and locomotion. J Neurosci 35:14476-14490.

Yokoi A, Hirashima M, Nozaki D (2011) Gain field encoding of the kinematics of both arms in the internal model enables flexible bimanual action. J Neurosci 31:17058-17068.

Yttri EA, Wang C, Liu Y, Snyder LH (2013) The parietal reach region is limb specific and not involved in eye-hand coordination. J Neurophysiol 111:520-532. 